\title{
TITLE
}

\section{Endothelial FOXC1 and FOXC2 promote intestinal regeneration after ischemia-reperfusion injury}

\section{Authors}

Can Tan, ${ }^{1}$ Pieter R. Norden, ${ }^{1}$ Ting Liu, ${ }^{1}$ Naoto Ujiie, ${ }^{1}$ Xiaocai Yan, ${ }^{2}$ Kazushi Aoto, ${ }^{3}$ Sagrario Ortega, ${ }^{4}$ Isabelle G. De Plaen, ${ }^{2}$ and Tsutomu Kume ${ }^{1 *}$

\section{Affiliations}

${ }^{1}$ Feinberg Cardiovascular and Renal Research Institute, Department of Medicine, Feinberg School of Medicine, Northwestern University, Chicago, Illinois, USA ${ }^{2}$ Department of Pediatrics, Feinberg School of Medicine, Northwestern University, Chicago, Illinois, USA

${ }^{3}$ Department of Biochemistry, Hamamatsu University School of Medicine, Hamamatsu, Japan

${ }^{4}$ Mouse Genome Editing Unit, Biotechnology Program, Spanish National Cancer Research Centre, Madrid, Spain

Correspondence to:

*Tsutomu Kume, Feinberg Cardiovascular and Renal Research Institute, Department of Medicine, Northwestern University School of Medicine, 300 E. Superior Street, Chicago, Illinois 60611, USA

Phone: 312.503.0623; E-mail: t-kume@northwestern.edu 


\begin{abstract}
Intestinal ischemia induces mucosal damage while simultaneously activating intestinal stem cells (ISCs), which subsequently regenerate the damaged intestinal epithelium. However, whether angiocrine factors secreted from vascular endothelial cells (ECs) - blood and lymphatic ECs (BECs and LECs, respectively) - regulate ISC-mediated regeneration have yet to be elucidated. Here, we identify FOXC1 and FOXC2 as essential regulators of angiocrine signaling in regeneration of the small intestine after ischemia-reperfusion (I/R) injury. EC- and LEC-specific deletions of Foxc1, Foxc2, or both in mice augment I/Rinduced intestinal damage by causing defects in vascular regrowth, expression of the chemokine CXCL12 and the Wnt activator R-spondin 3 in BECs and LECs, respectively, and activation of Wnt signaling in ISCs. Treatment with CXCL12 and R-spondin 3 rescues the I/R-induced intestinal damage in EC- and LEC-Foxc mutant mice, respectively. This study provides evidence that $\mathrm{FOXC1}$ and $\mathrm{FOXC} 2$ are required for intestinal regeneration by stimulating angiocrine CXCL12 and Wnt signaling.
\end{abstract}




\section{Introduction}

Tissue regeneration and repair is essential for maintaining physiological homeostasis and relies on the precise control of molecular networks that regulate, or are regulated by, the vasculature. ECs present in the blood and lymphatic vessels are crucial participants in the vascular-dependent processes that restore damaged tissue because they control the secretion of paracrine factors from both the vessels themselves and nearby cells $(1,2)$. However, the fundamental mechanisms by which the vascular system regulates tissue regeneration and repair are poorly understood. Thus, an adequate understanding of the biological processes that contribute to EC-dependent tissue repair, as well as their roles in the pathogenesis and potential treatment of vascular disease or injury, is crucially dependent on a thorough characterization of how blood/lymphatic vessels control tissue regeneration.

Intestinal ischemia is a life-threatening vascular emergency and can be caused by thrombus formation in the mesenteric vasculature, embolisms that arise as a consequence of cardiopulmonary disease, and disease- or shock-induced declines in perfusion, as well as when blood flow is interrupted by intestinal transplantation $(3,4)$. Moreover, impairments in intestinal microvasculature development contribute to the pathogenesis of neonatal necrotizing enterocolitis (NEC), which is the most common life-threatening gastrointestinal emergency in neonatal patients $(5,6)$. Both homeostasis and repair of the small intestine are mediated via intestinal stem cells (ISCs) $(7,8)$. Active ISCs express the specific marker leucine-rich repeat-containing $G$ protein-coupled receptor $5(\operatorname{Lgr} 5)$ and are located at the base of the crypts of the small intestine where they vigorously proliferate to 
continuously regenerate the intestinal epithelium. Conversely, a subpopulation of basalcrypt $\operatorname{Lgr} 5^{+}$cells are slow cycling and minimally proliferative $(8,9) . \mathrm{I} / \mathrm{R}$ injury, as well as radiation injury and stresses such as acute inflammation, induce apoptosis in proliferating $\operatorname{Lgr}^{+}$ISCs $(10,11)$, which subsequently regenerate the damaged intestinal epithelium. However, the mechanisms that coordinate the role of intestinal ECs in intestinal regeneration and repair, and whether components of the vascular system in the ISC niche such as BECs and LECs regulate the regenerative activity of ISCs as well as the preservation of the ISC niche after injury, have yet to be fully elucidated.

In adult mice, the proliferation of active ISCs is controlled, in part, by $\mathrm{Wnt} / \beta$-catenin signaling $(12,13)$, and canonical $\mathrm{Wnt} / \beta$-catenin signaling is promoted by the cooperative activity of Wnt proteins and R-spondins (RSPO1-RSPO4). Notably, Lgr5 functions (with Lgr4 and Lgr6) as a cognate receptor for R-spondins, and R-spondins are expressed in mesenchymal stromal cells of the ISC niche $(14,15)$. Among these mesenchymal stromal cells, Pdgfra ${ }^{\mathrm{lo}} \mathrm{Grem} 1+$ trophocytes are considered an essential source of R-spondins (RSPO1-RSPO3, especially RSPO3) (16), while Fox11+ mesenchymal cells (telocytes) and other non-epithelial stromal cells are believed to be an essential source of Wnt ligands (e.g., Wnt2b, Wnt4, and Wnt5a) in the ISC niche $(14,17-19)$. Importantly, both the number of $\operatorname{Lgr}^{+}$ISCs and the regenerative response to intestinal radiation injury are reduced by cotreatment with R-spondin 2- and R-spondin 3-neutralizing antibodies (20), and the mucosal damage induced by intestinal $\mathrm{I} / \mathrm{R}$ injury can be rescued by treatment with $\mathrm{R}$ spondin 3 (21). More importantly, intestinal RSPO3 $(15,16,22)$ and Wnt2 (16) are highly produced by LECs. 
The CXC chemokine CXCL12, also called stromal cell-derived factor 1 (SDF-1), is a homeostatic chemokine that expressed in many cell types such as stromal cell, ECs, fibroblasts in various tissues (23). CXCL12 is an essential factor for angiogenesis which involves the proliferation and migration of the endothelial cells to form neo-vessel networks (24). CXCL12 can be induced by hypoxic stress $(25,26)$ and regulates angiogenesis in an autocrine/paracrine manner by interacting with CXCR4 and CXCR7 $(27,28)$. In the small intestine, CXCR4 is expressed in the crypt epithelial cells $(29,30)$, and whereas CXCR4 deficiencies in the intestinal epithelium impair re-epithelialization after acute dextran sodium sulfate (DSS)-induced injury in mice (30), CXCL12overexpressing mesenchymal stem cells (MSCs) improve survival and intestinal epithelial recovery when administered to mice after radiation injury (29). Notably, CXCL12overexpressing MSCs are distributed in the villous stroma of the small intestine in irradiated mice, and local CXCL12 expression stimulates CXCR4 signaling in the intestinal crypts by activating $\beta$-catenin (29). Thus, CXCL12-CXCR4 signaling and the canonical Wnt/ $\beta$-catenin pathway appear to interact during intestinal regeneration.

FOXC1 and FOXC2 are closely related members of the FOX transcription factor family and have numerous essential roles in cardiovascular development, health, and disease, including the formation of ECs $(31,32)$. Mutations or changes in the copy number of human FOXCl are associated with autosomal-dominant Axenfeld-Rieger syndrome (ARS), which is characterized by abnormalities in the eye and extraocular defects (33), while inactivating mutations of human $F O X C 2$ are responsible for the autosomal dominant 
syndrome Lymphedema-distichiasis, which is characterized by obstructed lymph drainage in the limbs, venous valve failure, and the growth of extra eyelashes (distichiasis) (34). There is also some evidence that Foxc2 haploinsufficiency in mice increases their susceptibility to DDS-induced colitis (35); however, the precise function of FOXC1 and FOXC2 in vascular repair and intestinal regeneration after ischemic injury have yet to be determined.

In this study, we investigated whether FOXC1 and FOXC2 in intestinal BECs and LECs contributes to vascular repair and intestinal regeneration after $\mathrm{I} / \mathrm{R}$ injury by regulating paracrine signaling. To this end, we generated EC- and LEC-specific, single and compound mutant mice for Foxc1 and Foxc2. The Foxc mutations impair regeneration of the small intestine after I/R injury, accompanied by (1) defective repair of intestinal BECs and LECs, (2) reduced expression of CXCL12 and R-spondin 3 in intestinal BECs and LECs, respectively, and (3) decreased activation of the Wnt/ $\beta$-catenin pathway in ISCs. Most importantly, treatment with either CXCL12 or R-spondin 3 partially rescues the defects in intestinal repair and regeneration associated with EC- and LEC-Foxc1/c2 deficiency. CXCL12 enhance vascular repair, and both factors stimulate canonical Wnt/ $\beta$-catenin signaling, which subsequently increase the proliferation of intestinal epithelial cells. Together, our data show a new role for FOXC1 and FOXC2 as key mediators of angiocrine signaling in the intestinal blood/lymphatic vasculature during post-ischemic intestinal repair/regeneration, and our findings may have important implications for other ischemic conditions that are associated with impairments in tissue regeneration. 


\section{Results}

\section{Expression of Foxc1 and Foxc2 in the murine intestine}

We first evaluated the expression of Foxc1 and Foxc2 in intestinal BECs and LECs via quantitative real-time RT-PCR (qPCR) of CD45-CD31 $1^{+} \mathrm{ECs}$, which include both BECs and LECs isolated from the small intestine in adult mice (Fig. 1A). Notably, Foxcl was more highly expressed than Foxc2 in adult intestinal ECs. Intestinal ECs are particularly vulnerable to I/R injury, because they undergo apoptosis in response to oxidative stress (3); therefore, we investigated whether I/R injury alters Foxcl/c2 expression in the small intestine of adult mice. Intestinal I/R injury (36) was induced by clamping the superior mesenteric artery for 30 minutes to induce occlusion; then, the clamp was removed, and the small intestine was allowed to re-perfuse before the animal was sacrificed and the small intestine was explanted. Foxc1/Foxc2 expression was significantly greater in the intestinal $\mathrm{CD} 45^{-} / \mathrm{CD} 31^{+} \mathrm{ECs}$ of mice 4 hours after I/R injury than in the intestinal ECs of shamoperated animals (Fig. 1, B and C). These results are consistent with work in other labs showing that FOXC2 expression in renal tubular cells of the cortex and outer medulla also increases 24 hours after kidney I/R injury (37). Comparison of the relative levels of Foxc1 and Foxc 2 expression in the intestinal ECs 4 hours after I/R injury (Fig. 1D) compared with those under no surgery (Fig. 1A) revealed that both Foxc1 and Foxc2 were increased to a similar extent after I/R injury.

To investigate the expression pattern of FOXC1 in the adult mouse intestinal ECs, we performed whole-mount intestinal immunostaining labeled with CD31 (EC marker), LYVE1 (LEC marker) and FOXC1. In villus, FOXC1 was hardly detectable in both BECs 
and LECs, while in submucosa, FOXC1 was weakly expressed in the nuclei of BECs, but was still hardly detectable in submucosal LECs (Fig. 1E, no surgery). To investigate Foxc2 expression pattern in the intestinal vasculature, we crossed tamoxifen-inducible Foxc2$C r e^{E R T 2}$ knock-in mice (38) with dual Rosa26mTmG reporter mice (39). We used neonatal intestine because the adult expression of FOXC2 was too low to be detected by immunohistochemistry staining (IHC) (Fig. S4A, sham). The neonatal offspring were subsequently treated with tamoxifen from postnatal day $1(\mathrm{P} 1)$ to $\mathrm{P} 5$ and the distribution of Cre-recombined (EGFP+), Foxc2-expressing cells were assessed at P13 in the small intestine. We found that FOXC2-EGFP was highly expressed in LECs and weakly detected in both BECs and the intestinal muscular layer compared to the Cre negative control littermates (Fig. S1).

Expression patterns of FOXC1 and FOXC2 in the intestine were also examined in a mouse NEC model (40), which includes initial orogastric inoculation of neonatal mice with a standardized adult mouse commensal bacteria preparation and lipopolysaccharide (LPS) to perturb the normal intestinal colonization process, gavage with formula every 3 hours, and exposure to brief episodes of hypoxia for 1 min followed immediately by cold stress (10 min at $4^{\circ} \mathrm{C}$ ) twice daily. With this protocol, about $50-70 \%$ of mice typically develop between 36-72 hours intestinal injuries ranging from epithelial injury to transmural necrosis (40). At 24 hours after the neonates were subjected to the NEC protocol, immunohistochemical analyses of BEC (CD31 and endomucin [ECMN]) and LEC (PROX1) markers revealed that levels of FOXC1 and FOXC2 expression were increased in intestinal BECs and LECs (Fig. S2 and S3), compared to dam-fed littermate controls. 
Four hours after I/R injury, FOXC1 and FOXC2 protein levels were also upregulated in both intestinal BECs and LECs (Fig. 1E and Fig. S4A). Consistently, Foxc2-expressing, recombined EGFP+ cells were also detected in lacteals and villous capillaries of small intestines in adult $m T m G /+; F o x c 2-C r e^{E R T 2}$ mice after intestinal I/R injury (Fig. S4, B and C).

\section{Generation of tamoxifen-inducible, EC-specific, Foxc1/c2-mutant mice in the adult}

Murine Foxc1 and Foxc2 are both required for vascular development (41-43), but attempts to determine how the two genes function during postnatal and pathological (lymph)angiogenesis have been generally unsuccessful, because global single $\left(\right.$ Foxcl $^{-/-}$or

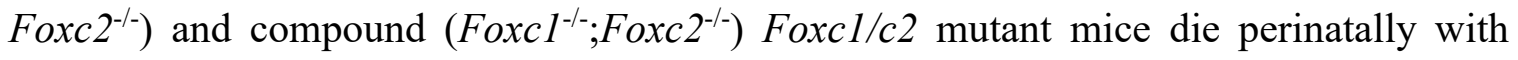
severe cardiovascular abnormalities (42). Therefore, we crossed conditional-null Foxclfl and Foxc $2^{f l}$ mutant mice (44) with $C d h 5-C r e^{E R T 2}$ mice (45) to generate tamoxifen-inducible, EC-specific, compound Foxc1;Foxc2-mutant $\left(C d h 5-C r e^{E R T 2} ; F_{o x c} 1^{f l f l} ; F_{o x c} 2^{f l f l}\right)$ mice, which (after the mutation is induced) are referred to as EC-Foxc-DKO mice (46). To induce the mutations, adult mice were treated with tamoxifen $(150 \mathrm{mg} / \mathrm{kg})$ by oral gavage for 5 consecutive days, and 12 days after tamoxifen treatment was completed (Fig. 1F), qPCR and immunohistochemical analyses confirmed that Foxc1- and Foxc2-expression was significantly reduced in intestinal ECs of EC-Foxc-DKO mice than in the corresponding cells of their control littermates (Fig. 1, G-I). Importantly, the small intestines of EC-FoxcDKO mice appeared morphologically normal 12 days after tamoxifen treatment (Fig. S4D), suggesting that EC expression of Foxcl and $F o x c 2$ is not required for maintaining intestinal 
epithelium homeostasis. Furthermore, since our mutants express Cre from the EC-specific Cdh5 promoter (45), we crossed them with dual Rosa26mTmG reporter mice (39) and then treated their adult offspring with tamoxifen as described above and identified recombined EGFP $^{+}$cells in the small intestines to confirm the efficiency of Cre-mediated recombination in intestinal BECs and LECs (Fig. S5A).

\section{EC-specific deletion of Foxc1 and Foxc 2 decreases intestinal mucosal recovery after}

\section{$\mathbf{I} / \mathbf{R}$}

When intestinal I/R injury was induced 12 days after tamoxifen treatment in adult mice (Fig. 2A), tissue from the distal jejunum was histologically graded according to the Chiu scoring system (47). The Chiu scores were then quantified 24 hours after I/R injury. Compared to the control mice, the intestinal mucosa remained severely injured in EC-FoxcDKO mice (Fig. 2, B and C). To further characterize how the loss of EC-specific Foxc1/c2 expression affects repair of the intestinal mucosa during recovery from $\mathrm{I} / \mathrm{R}$ injury, we also conducted a set of experiments in mice carrying tamoxifen-inducible, EC-specific mutations of each individual gene (i.e., EC-Foxc1-KO and EC-Foxc2-KO mice) and in their control littermates. Recovery from intestinal damage was impaired in both EC-Foxcl$\mathrm{KO}$ and EC-Foxc2-KO mice 24 hours after I/R injury (Fig. 2, D and E). As the extent of intestinal injury in the EC-specific Foxc single mutant mice was less than that in the double Foxc mutant mice, EC-Foxc1 and -Foxc2 expression is required for intestinal epithelial regeneration. Since I/R-induced local inflammatory response is critically associated with intestinal damage $(21,48)$, mRNA levels of proinflammatory regulators (TNF $\alpha, I L-6$ and Cox2) were measured 3 hours after I/R injury via qPCR. Expression levels of $T N F \alpha$ and 
Cox2 were significantly higher in the EC-Foxc-DKO mice than in the control mice (Fig. 2F), whereas increased $I L-6$ expression in EC-Foxc-DKO mice exhibited a trend toward significance $(P=0.2014)$. Furthermore, EC-specific loss of Foxc genes significantly reduced the proliferative response of intestinal epithelial cells to $\mathrm{I} / \mathrm{R}$ injury as assessed via immunostaining of EpCAM (intestinal epithelial cell marker) and BrdU (proliferative marker) (Fig. 2G), as well as the quantification of BrdU positive epithelial cell number per crypt (Fig. 2H).

\section{Wnt signaling in the small intestine is impaired in EC-Foxc-DKO mice after $\mathbf{I} / \mathbf{R}$} injury

$\beta$-catenin regulates the maintenance and regeneration of intestinal epithelial cells $(12,13)$ by translocating from the cytosol to the nucleus of ISCs and altering gene expression in response to activation of the canonical Wnt signaling pathway. This mechanism is consistent with our observations in control mice, because although $\beta$-catenin was located at the adherens junctions of epithelial cells in the villus, nuclear $\beta$-catenin was detected in ISCs co-immunostained with the ISC marker OLFM4 (49) at the crypt base (Fig. 2I, arrows) after I/R injury (18). However, nuclear localization of $\beta$-catenin was impaired and the number of cells that expressed the Wnt target cyclin D1 (CCND1) (18) was significantly reduced in the crypt of EC-Foxc-DKO mice both 24 and 48 hours after I/R injury (Fig. 2J and Fig. S6A). Together, the loss of EC-specific Foxc1/c2 expression impedes I/R-induced Wnt signaling in ISCs. 


\section{Defective mucosal recovery in LEC-specific Foxc mutant mice after intestinal I/R injury}

As $C d h 5$ is expressed in both the blood and lymphatic vasculatures, we generated mice carrying a tamoxifen-inducible, LEC-specific, compound homozygous Foxcl $^{-/-} ; F_{\text {oxc }} 2^{-/-}$ mutation (referred to herein as LEC-Foxc-DKO mice) by breeding conditional-null Foxc $1^{f l f l}$ and $F o x c 2^{f l f l}$ mice (44) with LEC-specific Vegfr3-Cre $e^{E R T 2}$ mice (50) to investigate intestinal LEC-specific functions of Foxcl and Foxc2 during I/R. We then confirmed Vegfr3-Cre-mediated recombination limited to intestinal LECs of adult LEC-Foxc-DKO mice crossed with the Rosa26mTmG reporter mice (Fig. S5B). The LEC-Foxc-DKO mice and their littermate control mice were subjected to the sham or I/R injury procedures, and LEC-specific deletion of Foxcl and Foxc 2 significantly increased the severity of intestinal mucosa injury 24 hours after I/R (Fig. 3A). Furthermore, increased intestinal damage was also noted in single LEC-Foxc1-KO and LEC-Foxc2-KO mice after I/R (Fig. 3, B and C), suggesting that both FOXC1 and FOXC2 are required in intestinal LECs for intestinal repair in response to $\mathrm{I} / \mathrm{R}$ injury. In addition, proliferating (BrdU+) intestinal epithelial cells were quantified in the crypts of the LEC-Foxc-DKO mice 24 hours after I/R injury, and the LEC-specific loss of Foxc1/c2 reduced the proliferative response of intestinal epithelial cells to I/R injury (Fig. 3D). Consistent with the increased severity of intestinal mucosa defects in the LEC-Foxc-DKO mice (Fig. 3A), the activation of Wnt signaling (i.e., nuclear localization of $\beta$-catenin, Fig. 3F) in ISCs and the number of Cyclin D1+ (CCND1+) cells per crypt after intestinal I/R injury were both reduced in the LEC-Foxc-DKO mice compared to the control mice (Fig. 3E and Fig. S6B). 


\section{Defective vascular recovery in EC- and LEC-specific deletions of Foxc1/c2 genes after intestinal $\mathbf{I} / \mathbf{R}$ injury}

The vascular recovery of villus BECs and LECs after I/R injury proceeds via a stepwise process in which blood capillaries (BECs) regrow earlier than lacteals (LECs) in the villous stroma (51). We found that the EC-Foxc-DKO mutation was associated with defective vascular repair of intestinal BECs and LECs after I/R injury (Fig. 4). Vascular endothelial growth factor (VEGF) receptor (R) 2 (VEGFR2) and VEGFR3 were highly expressed in the growing tips of villous BECs and LECs, respectively, from control mice after intestinal I/R injury (Fig. 4, A and B) but were severely diminished in EC-Foxc-DKO mice (arrows in Fig. 4, A-B, Movies S1 and S2). Notably, proliferation of intestinal BECs and LECs was also significantly reduced (Fig. 4, C-E), while the number of apoptotic BECs and LECs was significantly increased (Fig. 4, F-H), in the EC-Foxc-DKO mice than in their control mice after I/R injury. Whole-mount immunostaining for CD31 and LYVE1 was performed (Fig. S7, A and B), and the length of blood capillaries and lacteals (Fig. 4I) as well as the percentage of lacteal length to blood capillary length (Fig. 4J) were measured. The regrowth of both blood capillaries and lacteals in the villi were significantly decreased in the EC-Foxc-DKO mice after intestinal I/R injury, whereas EC-Foxc-double mutant lacteals were shorter than controls in sham treatments. Similarly, shorter lacteals were found in proximal jejunums in EC-Foxc-DKO neonatal mice at P7 after Tm treatment from P1 to P5 (Fig. S7, D-F), suggesting that Foxc1/c2 are required for the maintenance of lacteal length. Furthermore, whole-mount immunostaining for VEGFR2 (Fig. S7C) and subsequent quantification reveal a reduction in branches (between branching points) (Fig. 4K) and branching points (Fig. 4L) of blood capillaries in the EC-Foxc-DKO mice after 
intestinal I/R injury. Taken together, these results suggest that EC-Foxc1/c2 expression contributes to the repair of ischemic intestinal mucosa by promoting the recovery of BECs and LECs.

\section{R-spondin 3 and Cxcl12 expression after $\mathbf{I} / \mathrm{R}$ injury is lower in intestinal LECs and}

\section{BECs from EC-Foxc-DKO mice than from control mice, respectively}

To investigate molecular mechanisms associated with impaired intestinal regeneration in EC-Foxc-DKO mutants following $\mathrm{I} / \mathrm{R}$, we performed single-cell RNA sequencing (scRNA-seq) analyses of distal jejuna from control and EC-Foxc-DKO mice 18.5 hours after I/R injury. Dimensionality reduction and clustering analysis identified 22 transcriptionally distinct cell clusters (Fig. 5A and Fig. S8A) based on known gene markers for each specific cell type (Supplemental Table 1), including BECs, LECs, stromal cells, epithelial cells and other cell types. When interpreted according to the recent classification of stromal cell populations in the ISC niche (15), the results from our scRNA-seq experiments indicates that $R$-spondin 3 (Rspo3) is mainly expressed in two clusters (LECs and Telocytes/Trophocytes) in intestine $18.5 \mathrm{~h}$ after intestinal I/R injury (Fig. 5B, cluster 12 and 20, respectively). Low expression of Rspo3 was found in the cluster of myocytes/pericytes (Fig. 5B, cluster 17). Further sub-clustering performed on the cluster of Telocytes/Trophocytes based on their known markers (52) identified 3 cell clusters, Trophocytes, Pdgfra ${ }^{l o}$ Cd81- stromal cells, and Telocytes (Fig. S8B). A dot plot used for visualizing differential gene expression (mean expression level) as well as gene expression frequency in different cell clusters (Fig. 5C) showed that Rspo3 was decreased in both LECs and Trophocyte clusters in EC-Foxc-DKO intestines compared with controls after 
I/R injury. In Trophocyte cluster, the moderately decreased trend of Rspo3 was not significant (Fig. S8C). Similar Rspo3 levels were found in the other 3 cell clusters between the two groups. Since the percentage of the LECs captured by scRNA-seq was very low $(0.373 \%$, Fig. S8A), the number of LECs obtained and used for analysis was very limited, and the decrease of Rspo3 expression in LECs was not significant (Fig. S8D). However, by IHC, RSPO3 protein was lower in intestinal LECs from EC-Foxc-DKO mice than control mice 24 hours after I/R injury (Fig. 5D).

The results from our scRNA-seq experiments also identified numerous genes that were differentially expressed in intestinal BECs from EC-Foxc-DKO and control mice after I/R injury (Fig. 5E), including the anti-angiogenic factor Adamts1 $(53,54)$ and the CXC chemokine Cxcl12, which were up- and down-regulated, respectively, in EC-Foxc-DKO BECs. Notably, although Cxcl12 was also expressed in the Telocyte/Trophocyte and Fibroblast cell clusters (Fig. 5, F and G), it was significantly downregulated only in BECs (Fig. 5, E and G), which have a larger cell population than the other two cell clusters (Fig. 5F and Fig. S8A). Furthermore, qPCR analysis validated that Cxcl12 expression was significantly reduced in isolated intestinal $\mathrm{CD}^{-} / \mathrm{CD} 31^{+} \mathrm{ECs}$ of both sham- and I/Rtreated EC-Foxc-DKO mice compared to the control mice (Fig. 5H).

\section{Treatment with $\mathrm{R}$-spondin 3 rescues the defective repair of small intestines in $\mathrm{EC}$ - and LEC-Foxc-DKO mice after I/R injury}

RSPO3 prevents I/R-induced intestinal tissue damage and vascular leakage (21), and RSPO3 is a key regulator of Wnt signaling during intestinal regeneration $(20,21)$. 
Accordingly, we sought to examine whether RSPO3 treatment can rescue the defective repair of small intestines in EC-Foxc-DKO mice following I/R injury. Adult EC-FoxcDKO mice were randomly assigned to two experimental groups and were treated with PBS or RSPO3 $(5 \mu \mathrm{g}$ in $100 \mu \mathrm{L}$ PBS per mouse) by retro-orbital injection $30 \mathrm{~min}$ before intestinal ischemia. Quantification of Chiu scores was then performed 24 hours after I/R injury. Intriguingly, RSPO3 treatment partially rescued the defective repair of small intestines in EC-Foxc-DKO mice (Fig. 6, A and B). RSPO3 is expressed in LECs of the small intestine, but not in BECs (Fig. 5B). To further define the specific roles of LECFoxc1/c2 expression in the RSPO3-dependent rescue of intestinal repair, equivalent experiments were performed with adult mice carrying LEC-specific mutations of both Foxc genes. Similar rescue effects of the RSPO3 treatment were observed in LEC-Foxc-DKO intestines 24 hours after I/R injury with improvement of the Chiu score to the level similar to I/R-exposed control mice (Fig. 6, C and D). Thus, treatment with RSPO3 is sufficient to fully recover intestinal I/R injury in LEC-specific Foxc 1/c2 deficient mice.

Immunoglobulin $\mathrm{M}$ (IgM) and complement also contribute to intestinal I/R injury (21), but RSPO3 treatment suppresses the deposition of IgM and complement in damaged intestinal tissue (21), likely by enhancing the activation of Wnt signaling in ISCs of the small intestine. Therefore, IgM deposition was evaluated in damaged intestines of control and EC-Foxc-DKO mice 24 hours after I/R injury via immunostaining (Fig. S9). Compared to control mice, IgM deposition was increased in EC-Foxc-DKO mice, whereas administration of RSPO3 reduced the level of IgM in EC-Fox-DKO mice. The levels of $\operatorname{IgM}$ deposition were further quantified via western blotting $(21,36)$. I/R-enhanced IgM 
levels in the intestinal tissues were greater in EC-Foxc-DKO mice compared to controls

(Fig. 6, E and F). More importantly, RSPO3 treatment significantly reduced the levels of $\operatorname{IgM}$ in the EC-Foxc-DKO mice after I/R injury (Fig. 6, G and H). Collectively, these observations demonstrate that Foxc1 and Foxc2 expression in the intestinal vasculature contributes to intestinal mucosal recovery and regeneration after I/R injury.

\section{Treatment with CXCL12 partially rescue the defective repair of small intestines in}

\section{EC-Foxc-DKO mice after I/R injury}

Cxcl12 was only downregulated in intestinal BECs, but not in the Telocyte/Trophocyte and

Fibroblast cell clusters of EC-Foxc-DKO mouse intestine after I/R injury (Fig. 5, E and G). Given evidence that hypoxia regulates expression of CXCL12 $(25,26)$, a key regulator of angiogenesis $(27,28)$, we also sought to determine whether pre-treatment with CXCL12 can rescue the defects in the vascular regrowth and intestinal repair associated with ECFoxc1/c2 deficiencies. Adult EC-Foxc-DKO mice were treated with PBS or CXCL12 via retro-orbital injection as described previously (55), and I/R induction surgery was performed 30 minutes later. The degree of intestinal mucosal damage 24 hours after I/R injury was then quantified via the Chiu scoring system. The CXCL12 treatment partially rescued the defective repair of small intestines in the EC-Foxc-DKO mice (Fig. 7, A and B). Moreover, nuclear localization of $\beta$-catenin was observed in crypt ISCs of CXCL12pretreated EC-Foxc-DKO mice after I/R injury compared to the PBS-treatment (Fig. 7C), and the number of cells expressing the Wnt target cyclin D1 (CCND1) (18) was significantly increased in the crypt ISCs of EC-Foxc-DKO mice pretreated with CXCL12 after I/R injury (Fig. 7D). Administration of CXCL12 also improved the defective vascular 
recovery (CD31+ vessel density) of EC-Foxc-DKO mice 24 hours after I/R injury (Fig. 7, E and F). Therefore, CXCL12 treatment rescues the defects in vascular regrowth and intestinal repair associated with EC-Foxc1/c2 deficiencies by enhancing vascular recovery and stimulating Wnt signaling in the crypts of the intestinal epithelium.

\section{Discussion}

Little is known about the molecular mechanisms that regulate BEC/LEC-derived paracrine signaling in intestinal regeneration. In the present study, we show that EC-Foxc1/Foxc2 expression is crucial for repair of the intestinal mucosa, BECs, and LECs after I/R injury, and that the EC- and LEC-Foxc-DKO mutations in mice impair canonical $\mathrm{Wnt} / \beta$-catenin signaling in ISCs at the crypt base. Furthermore, our scRNA-seq data indicate that Rspo3 expression is attenuated in LECs and stromal cells of the EC-Foxc-DKO mice after intestinal $\mathrm{I} / \mathrm{R}$ injury, which is at least partially attributable to impairments in intestinal regeneration because the activity of ISCs appears to be crucially dependent on Wnt/ $\beta$ catenin signaling in subepithelial stromal cells of the $\operatorname{ISC}$ niche $(12,13)$. We also show that the chemokine CXCL12, an autocrine/paracrine factor of angiogenesis, is reduced in intestinal BECs of the EC-Foxc-DKO mice after I/R injury. Most significantly, pretreatment with RSPO3 and CXCL12 rescues the defective repair of small intestines in the LEC-Foxc-DKO and EC-Foxc-DKO mice, respectively. Consequently, this study elucidates the novel mechanisms that mediate the role of EC-specific Foxc expression in repair of intestinal mucosa and vasculature, regulation of paracrine signaling pathways, and activation of ISCs after intestinal I/R injury. 
Adult single and compound EC- (or LEC)-specific Foxc1/c2 mutant mice provide us with the first opportunity to comprehensively characterize how Foxc genes regulate the postischemic repair of intestinal blood/lymphatic vessels after $\mathrm{I} / \mathrm{R}$ injury and intestinal epithelial regeneration by modulating Wnt and chemokine CXCL12 signaling. Because the EC-Foxc-DKO and LEC-Foxc-DKO mutations are induced by different drivers (i.e., Cdh5-Cre $e^{E R T 2}$ and Vegfr3-Cre $e^{E R T 2}$ ), the extent of Foxc downregulation in the two mutant mouse lines may not be equal. However, the degree of impairments in intestinal repair is consistently greater in the EC-Foxc-DKO mutant line than in the LEC-Foxc-DKO mutant line, suggesting that Foxc1 and Foxc2 are required in both BECs and LECs for intestinal tissue repair. In addition to analyses of EC- (and LEC-) specific mutant mice for both Foxc1 and Foxc2, equivalent experiments were performed with adult mice carrying EC- (and LEC-) specific KO mutations of each individual Foxc genes to determine the similarities and differences between the phenotypes associated with each deletion. While our qPCR and scRNA-seq analyses show that expression levels of Foxcl in intestinal ECs are higher than those of Foxc2, the phenotypic differences between EC- (or LEC-) Foxc1-KO and EC- (or LEC-) Foxc2-KO mice are not particularly distinct. Although the reason(s) for the phenotypic similarities remains unclear, recent evidence indicates that Foxc 2 is essential for the maintenance of intestinal LECs, and that treatment with antibiotics to deplete gut microbiota rescues the phenotype of LEC-specific adult Foxc2 mutant mice, including lymphatic dilation in the small intestine (56). Bacterial translocation occurs when the intestinal barrier is disrupted by $\mathrm{I} / \mathrm{R}$ injury, and intestinal damage after $\mathrm{I} / \mathrm{R}$ injury can be attenuated by pretreating the animals with an antibiotic cocktail to deplete gut commensal bacteria (36). It remains to be elucidated whether commensal bacteria contribute to aberrant 
intestinal recovery and regeneration in our EC/LEC-specific Foxc mutant mice. Importantly, our results with the mouse NEC model, which includes orogastric inoculation with a standardized adult mouse commensal bacteria preparation and lipopolysaccharides (LPS) to perturb the normal intestinal colonization process, show that FOXC1 and FOXC2 levels are upregulated in intestinal ECs of neonatal mice (Fig. S2 and S3). Therefore, they may also be involved in the pathogenesis of NEC in humans.

The results from this study demonstrate that the EC-specific mutations of both Foxc genes in mice impair the regulation of Rspo3 in LECs (Fig. 5, C and D), and that RSPO3 treatment rescues the defective repair of small intestines in both EC-Foxc-DKO and LEC-Foxc-DKO mice after I/R injury (Fig. 6). These observations are consistent with reports that RSPO3 prevents I/R-induced intestinal tissue damage (21), and that RSPO3 is a key regulator of Wnt signaling during intestinal regeneration $(20,21)$. While intestinal RSPO3 is known to be produced by LECs $(15,22)$, we demonstrate that pretreatment with RSPO3 almost completely rescues the impaired intestinal repair in the LEC-Foxc-DKO mice, implicating a paracrine effect of LEC-mediated RSPO3 signaling on the activation of ISCs after I/R injury. We analyzed data deposited in the ENCODE database (57) with HOMER (Hypergeometric Optimization of Motif EnRichment) software (58), as recently described in our analysis of the PRICKLE1 locus (46), and identified putative FoxC binding sites (RYMAAYA or RYACACA) in the vicinity of the human RSPO3 locus (Fig. S10A and Methods). These sites are conserved between human and mouse, and contain histone methylated and acetylated regions (e.g., H3K4Me1 and H3K27Ac ChIP peaks), DNaseI hypersensitive regions, and transcriptionally active regions (identified by Transcription 
Factor ChIP-seq data). Thus, FOXC1 and FOXC2 are likely to regulate $R S P O 3$ expression directly in LECs. Paracrine RSPO3/Wnt signaling also plays a role in protecting hepatocytes against I/R injury (59), and RSPO3 produced by lung ECs in response to inflammatory injury stimulates lung interstitial macrophages in a paracrine manner $(60)$. Accordingly, it is conceivable that RSPO3 is a critical paracrine regulator in the regulation of tissue repair under pathological settings.

The chemokine CXCL12 is an angiocrine factor that is secreted from BECs and regulates organ-specific tissue repair, regeneration, and homeostasis, including the liver and bone marrow $(2,61,62)$. In the lung, autocrine signaling of CXCL12/CXCR4 in pulmonary ECs stimulates endothelial proliferation contributing to the pathogenesis of pulmonary arterial hypertension (63). However, the function of EC-derived CXCL12 paracrine/autocrine signaling in damaged organs after injury remains largely unknown. The results from our study demonstrate that $\mathrm{Cxcl12}$ expression is downregulated in the intestinal BEC cluster and intestinal CD45-/CD31+ ECs of the EC-Foxc-DKO mice after I/R injury (Fig. 5), and that CXCL12 treatment partially rescues the defective repair of small intestines in ECFoxc-DKO mice after I/R injury (Fig. 7). Our gene expression analyses are in accord with recent evidence that FOXC1 controls CXCL12 expression in CXCL12-abundant reticular (CAR) progenitors and Schwann cells $(64,65)$. Whether FOXC1 and FOXC2 directly regulate CXCL12 expression in BECs is unknown, however we identified putative FOXC binding sites (RYMAAYA or RYACACA) (66-68) in evolutionarily conserved regions of the human CXCL12 locus (Fig. S10B and Methods). EC-derived CXCL12 promotes angiogenesis via an autocrine mechanism (28), and CXCL12-CXCR4 signaling cooperates 
with VEGF to enhance angiogenic processes such as the morphogenesis and sprouting of vascular tubes (69). Since VEGFR2 levels at the angiogenic front of growing blood capillaries in the villus are lower in EC-Foxc-DKO mice than in control mice after intestinal I/R injury (Fig. 4), it is likely that intestinal EC-derived CXCL12 autocrine signaling is defective in EC-Foxc-DKO mice. Consistently, impaired vascular recovery of the EC-Foxc-DKO mice after intestinal I/R injury is rescued by CXCL12 treatment (Fig. 7). The vascular regrowth of villus BECs and LECs after I/R injury proceeds via a stepwise, interactive process, and regeneration of villus blood vessels precedes reconstruction of lacteals (51) (Fig. 4, Movies S1 and S2). The severity of mucosal damage is greater in the EC-Foxc-DKO mice than in the LEC-Foxc-DKO mice, indicating the possible involvement of BEC-derived paracrine factor(s) in intestinal epithelial repair and regeneration. When the $\mathrm{CXCR} 4$ receptor in intestinal epithelial cells is activated by treatment with CXCL12-overexpressing MSCs, this paracrine (angiocrine) signaling pathway subsequently stimulates canonical Wnt/ $\beta$-catenin signaling and regulates the proliferation of ISCs (29). Notably, treatment with CXCL12 stimulated activation of Wnt signaling in the ISC of the EC-Foxc-DKO mice (Fig. 7). Therefore, our results demonstrate that FOXC1 and FOXC2 promote endothelial-derived CXCL12 signaling in intestinal ischemia, thereby controlling vascular regrowth and intestinal regeneration in an autocrine/paracrine manner.

In summary, our study demonstrates that $\mathrm{BEC} / \mathrm{LEC}-\mathrm{FOXC1/FOXC2}$ expression regulates the repair of the intestinal vasculature and mucosal damage during intestinal regeneration after I/R injury by controlling the R-spondin 3 and CXCL12 signaling pathways. Thus, 
FOXC1 and FOXC2 regulate molecular and cellular mechanisms that maintain blood/lymphatic vascular function in the small intestine, as well as vascular-mediated signaling to ISCs and the ISC niche during intestinal repair. Collectively, this study provides new insights into fundamental processes that are critically involved in recovery from ischemic disease and injury and may have important implications for the treatment of other ischemic conditions that are associated with impairments in tissue regeneration and stem-cell activity, including cardiovascular disease.

\section{Materials and Methods}

\section{Animal husbandry}

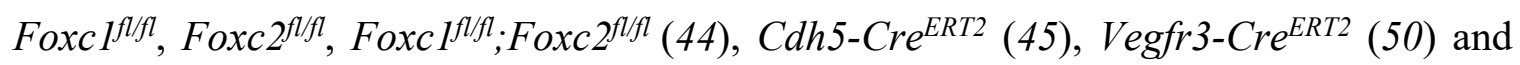

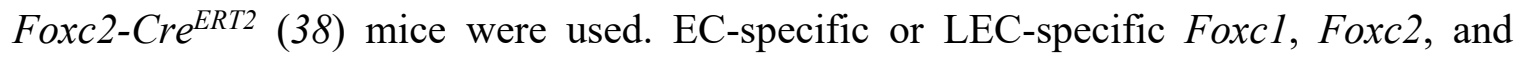
compound Foxc1;Foxc2 mutant mice were generated by crossing Foxc-floxed females $\left(F_{o x c} 1^{f l f l}, F_{o x c} 2^{f l f f l}\right.$, and Foxc1 $\left.1^{f l f l} ; F_{o x c} 2^{f l f l}\right)$ with $C d h 5-C r e^{E R T 2} ; F_{o x c} 1^{f l f l}(\mathrm{EC}-F o x c 1-\mathrm{KO})$, $C d h 5-C r e^{E R T 2} ; F_{o x c} 2^{f l f l} \quad(\mathrm{EC}-F o x c 2-\mathrm{KO}), \quad C d h 5-C r e^{E R T 2} ; F_{o x c} 1^{f l f l} ; F_{0 x c} 2^{f l / f l} \quad(\mathrm{EC}-F o x c-$

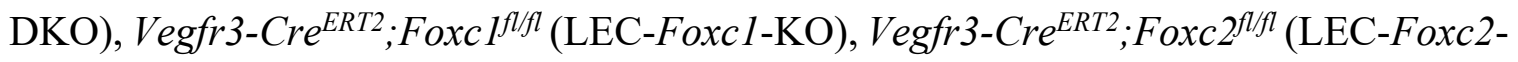
$\mathrm{KO}), \quad \operatorname{Vegfr} 3-C r e^{E R T 2} ; F_{\text {oxc }} 1^{f l f l} ; F_{0 x c} 2^{f l f l}$ (LEC-Foxc-DKO) males, respectively, as described previously (46). For Cre recombination efficiency detection, $m T m G /+$;Cdh5Cre $^{\text {ERT2 }} ;$ Foxc $^{f l f l} ; F_{\text {Oxc }} 2^{f l f l} \quad$ (mTmG/EC-Foxc-DKO), $\quad m T m G /+; V e g f r 3-$ $\operatorname{Cre}^{E R T 2} ; F_{\text {oxc }} 1^{f l f l} ; F_{\text {oxc }} 2^{f l f l}\left(\mathrm{mTmG} / \mathrm{LEC}-\right.$ Foxc-DKO) and $m T m G /+; F o x c 2-C r e^{E R T 2}$ mice

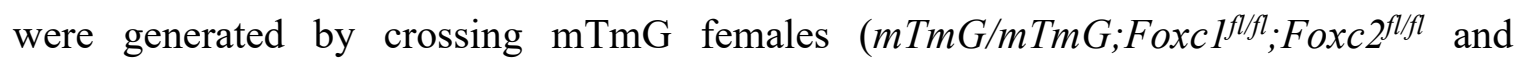

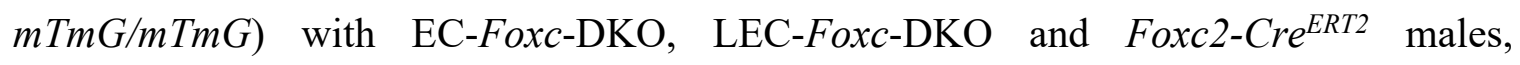
respectively. Genotyping of mice was performed by Transnetyx Inc. 


\section{Tamoxifen treatment}

For adult mice, Tamoxifen (Tm, Cayman chemical \#13258) was dissolved in corn oil (Sigma \#C8267) at a concentration of $40 \mathrm{mg} / \mathrm{mL}(70)$ by shaking at $37^{\circ} \mathrm{C}$ for $3-4$ hours. Aliquots of $\mathrm{Tm}\left(5\right.$ doses) were stored at $-20^{\circ} \mathrm{C}$ and pre-warmed at $37^{\circ} \mathrm{C}$ before each use for the duration of injections. 7-8-week-old male adult mice were treated with $150 \mathrm{mg} / \mathrm{kg}$ Tm by oral gavage once daily for 5 consecutive days. For neonatal mice, individuals were treated with $\operatorname{Tm}(75 \mu \mathrm{g})$ by oral gavage once daily from postnatal day $1(\mathrm{P} 1)$ to day $5(\mathrm{P} 5)$ (46).

\section{Cre recombination efficiency detection}

$\mathrm{mTmG} / \mathrm{EC}-$ Foxc-DKO, mTmG/LEC-Foxc-DKO and $m T m G /+; F o x c 2-C r e^{E R T 2}$ mice were used. The Cre negative $\mathrm{mTmG}$ mice were used as control. Twelve days after Tm treatment, the distal jejunum was harvested and fixed in $4 \%$ PFA at $4^{\circ} \mathrm{C}$ for $4 \mathrm{~h}$, dehydrated in $30 \%$ sucrose in PBS at $4^{\circ} \mathrm{C}$ for $\mathrm{O} / \mathrm{N}$, and embedded in Tissue-Tek O.C.T. Compound (Sakura Finetek, USA). 10 or $15 \mu \mathrm{m}$ cryosections were cut and immunostained with CD31 or LYVE1 antibody (Supplemental Table 2), counterstained with/without GFP antibody and a nuclear-specific dye DAPI. EGFP fluorescent signal was detected under Zeiss AxioVision fluorescent microscope to evaluate the Cre recombination efficiency.

\section{Mouse small intestinal ischemia and reperfusion (I/R) surgery}

Twelve days after Tm treatment, mice were subjected to small intestinal ischemia and reperfusion (I/R) surgery as previously described (36). Briefly, mice were anaesthetized with inhalation of Isoflurane. Adequate depth of anesthesia was assessed by lack of 
response to toe pinch. Body temperature was maintained throughout the procedures by placing animals on water circulating pads covered with sterile drape. The surgical field was maintained at all times through the use of aseptic technique. A midline laparotomy was performed, and the superior mesenteric artery (SMA) was then identified, isolated, and a small nontraumatic vascular clamp was applied to the SMA. Following clamp placement, the intestines was replaced into the abdominal cavity carefully. $500 \mu \mathrm{L}$ of $10 \mathrm{U} / \mathrm{ml}$ heparinized-saline was added directly into the peritoneal cavity via syringe to avoid coagulation of blood. The incision was then covered with well moistened gauze. After this ischemic phase for $30 \mathrm{~min}$, the clamp was removed, and the intestine was allowed to reperfuse. $500 \mu \mathrm{L}$ of sterile saline is administered to the peritoneal cavity to compensate for the fluid loss during surgery. A Chromic Gut Suture (4-0) was then used to close the muscle layer, followed by the closure of the skin with wound clips. Sham-operated mice were subjected to the exact same surgical procedure, aside from clamp placement.

\section{Histopathological evaluation of intestinal mucosal damage}

For Hematoxylin and Eosin (H\&E) staining of small intestine $24 \mathrm{~h}$ after intestinal $\mathrm{I} / \mathrm{R}$, distal jejunum was collected and fixed in $4 \%$ PFA at $4{ }^{\circ} \mathrm{C}$ for $\mathrm{O} / \mathrm{N}$. After the fixation, the intestine was washed 3 times with PBS and transferred into $70 \%$ ethanol for further tissue processing and paraffin embedding. $4 \mu \mathrm{m}$ paraffin sections were stained with Hematoxylin and Eosin. Based on the H\&E staining, Chiu Score (47) was used for evaluating the intestinal mucosal damage after I/R: grade 0, normal mucosa; grade 1, development of subepithelial Gruenhagen's space at the apex of the villus; grade 2, extension of the space with moderate epithelial lifting from the lamina propria; grade 3, massive epithelial lifting 
with a few denuded villi; grade 4, denuded villi with exposed lamina propria and dilated capillaries; and grade 5, digestion and disintegration of the lamina propria, hemorrhage and ulceration. Higher scores represent more severe damage.

\section{BrdU treatment}

Mice were treated with one dose of BrdU (Sigma \#B5002, $10 \mathrm{mg} / \mathrm{mL}$ in PBS, $5 \mathrm{mg} / \mathrm{kg}$ ) by intraperitoneal (i.p.) injection $2 \mathrm{~h}$ or $18.5 \mathrm{~h}$ (for evaluation of proliferative intestinal epithelium or proliferative BECs and LECs, respectively) before tissue dissection (at I/R24h and I/R-18.5h respectively).

\section{Tissue collection}

For histological analysis, transcardial perfusion was performed on the adult mice with cold PBS followed by 4\% paraformaldehyde (PFA) after anesthesia. Distal jejunum was selected for this study because this segment has the most severe mucosal damage after intestinal I/R surgery compared to other segments according to pilot experiments. Distal jejunum was harvested and cut longitudinally to expose the lumen. After several washes with PBS, the intestine was post-fixed in $4 \%$ PFA at $4^{\circ} \mathrm{C}$ for $4 \mathrm{~h}$ (for immunohistochemistry staining on frozen sections, IHC-F) or for overnight $(\mathrm{O} / \mathrm{N})$ (for $\mathrm{HE}$ staining, immunohistochemistry staining on paraffin sections, IHC-P). For qPCR and Western blot on whole tissue lysates of the small intestine, blood was removed by transcardial perfusion with cold PBS. Distal jejunum was harvested, opened longitudinally, and washed with cold PBS, then snap-frozen in liquid nitrogen for the following protocols. The tissue collection for neonatal intestinal whole-mount staining, the neonates were euthanized at P7 after Tm 
treatment from P1 to P5. The proximal jejunums were collected, washed with cold PBS, and fixed in $4 \% \mathrm{PFA}$ at $4^{\circ} \mathrm{C}$ for $4 \mathrm{~h}$, then subjected to the whole-mount staining protocol.

\section{Whole-mount (WM) staining}

Whole-mount staining of small intestine was performed as previously described (71). Briefly, Distal jejunum was dissected, pinned onto a silicon plate, and fixed with fixation solution $(0.5 \%$ PFA, $15 \%$ picric acid and $100 \mathrm{mM}$ phosphate buffer, $\mathrm{pH} 7.0)$ at $4{ }^{\circ} \mathrm{C}$ for O/N. Samples were washed with PBS, and subsequently dehydrated by $10 \%$ sucrose for 3 h, followed by $20 \%$ sucrose $+10 \%$ glycerol in $\mathrm{PBS}$ for $\mathrm{O} / \mathrm{N}$ at $4{ }^{\circ} \mathrm{C}$. After being washed with PBS, samples were incubated with blocking buffer (5\% donkey serum, $0.5 \%$ BSA, $0.3 \%$ Triton $\mathrm{X}-100,0.1 \% \mathrm{NaN}_{3}$ in $\mathrm{PBS}$ ) for $2 \mathrm{~h}$ at $4^{\circ} \mathrm{C}$, and then incubated with the indicated primary antibodies (Supplemental Table 2) diluted in the blocking buffer for $\mathrm{O} / \mathrm{N}$ at $4{ }^{\circ} \mathrm{C}$. Samples were washed with PBST (0.3\% Triton X-100 in PBS) for several times, followed by incubation with indicated fluorochrome-conjugated secondary antibodies (Supplemental Table 2) diluted in the blocking buffer for $\mathrm{O} / \mathrm{N}$ at $4^{\circ} \mathrm{C}$. The samples were washed again with PBST, post-fixed with 4\% PFA, cut into small strips (one or two villi wide), cleared with FocusClear (CelExplorer Labs \#FC-101) and mounted on slides in mounting medium.

\section{Immunohistochemistry (IHC) staining}

For IHC-P, 4 or $15 \mu \mathrm{m}$ paraffin sections were deparaffinized, rehydrated, subjected to antigen retrieval, permeabilized with PBST, blocked with blocking buffer containing 5\% Donkey serum in PBST for 30 min at room temperature (RT) and incubated with indicated 
antibodies (Supplemental Table 2) in blocking solution for $\mathrm{O} / \mathrm{N}$ at $4^{\circ} \mathrm{C}$. The sections were washed with PBS and incubated with indicated fluorochrome-conjugated secondary antibodies (Supplemental Table 2) in PBS for $1 \mathrm{~h}$ at RT. After several washes with PBS, the sections were counterstained with DAPI and mounted with mounting medium. For IHC-F, 10 or $15 \mu \mathrm{m}$ frozen sections were washed with PBS, and then subjected to the same blocking and antibody incubation protocols as IHC-P but without the step of antigen retrieval. For BrdU immunostaining, additional step of $2 \mathrm{~N} \mathrm{HCl}$ treatment is needed for the paraffin sections after the permeablization of TritonX-100 and before the blocking step. TUNEL staining was performed using In Situ Cell Death Detection Kit (Roche \#11684795910) according to the manufacturer's instructions.

\section{Imaging}

H\&E staining images were acquired using an Olympus Vanox AHBT3 Research Microscope (original magnification 100x, Tokyo, Japan). Fluorescent images were acquired using a Zeiss AxioVision fluorescence microscope or a Nikon A1 Confocal Laser Microscope with the software of Zeiss AxioVision SE64 Rel. 4.9.1 or NIS-Elements Viewer 4.20, respectively. Images were processed and analyzed with Adobe Photoshop, Imaris and Fiji (ImageJ) software. Imaris imaging software was used to create movies (Movies S1 and S2) for the 3-D structure of the blood and lymphatic vasculatures in villi after intestinal I/R.

\section{Rescue experiments}


In rescue experiments, mice were treated with $5 \mu \mathrm{g}$ RSPO3 (R\&D systems \#4120RS025CF) in $100 \mu \mathrm{L}$ PBS (21), or CXCL12 $\alpha$ (PeproTech \#250-20A) in PBS at a dose of $50 \mu \mathrm{g} / \mathrm{kg}$ of body weight (BW) (55) by retro-orbital injection $30 \mathrm{~min}$ before intestinal ischemia. Distal jejunum was harvested after I/R at $18.5 \mathrm{~h}(\mathrm{WB})$ or $24 \mathrm{~h}(\mathrm{H} \& \mathrm{E}$, IHC) for further analysis.

\section{Endothelial cell isolation from small intestine}

ECs were isolated from the distal jejunum for further qPCR analysis. Briefly, the single cell suspension was prepared as described in Supplemental Methods. The cell suspension was then incubated with magnetic Dynabeads (Invitrogen \#11035) labeled with CD45 antibody (Biolegend \#103102) to deplete the CD45+ cell population. The CD45- cell suspension was then incubated with Dynabeads labeled with CD31 antibody (BD \#553369) for the isolation of CD45-CD31+ cells. Finally, the CD45-CD31+ ECs (72) were collected for RNA isolation and qPCR analysis.

\section{RNA isolation and qPCR analysis}

For RNA extraction from isolated ECs, a RNeasy Mini Kit (Qiagen \#74104) was used according to the manufacturer's instructions. For isolation of RNA from whole distal jejunum, the tissue was grinned by using mortar and pestle chilled with liquid nitrogen. TRIzol $^{\mathrm{TM}}$ Reagent (Invitrogen \#15596026) was then used to isolate RNA according to the manufacturer's instructions. The concentration of RNA was determined using NanoDrop ${ }^{\text {TM }} 2000$ Spectrophotometers (Thermo Scientific). cDNA was synthesized using an iScript reverse transcriptase kit (Bio-Rad \#170-8891) according to the manufacturer's 
instructions. qPCR was performed on triplicates of cDNA samples by using QuantStudio®

3 Real-Time PCR System (Applied Biosystems), Fast SYBR reaction mix (Applied

Biosystems), and gene specific primer sets. $18 \mathrm{~S}$ was used as an internal standard for mRNA expression. Primer sequences are provided in Supplemental Table 3.

\section{Western Blot}

The frozen intestinal tissue was grinned using mortar and pestle chilled with liquid nitrogen, followed by lysis in RIPA buffer $(150 \mathrm{mM} \mathrm{NaCl}, 1 \%$ Nonidet P-40, $0.5 \%$ sodium deoxycholate, $0.1 \%$ SDS and 50mM Tris, $\mathrm{pH} 7.4$ ) containing protease inhibitors (Roche \#4693116001). After centrifugation, the supernatant of the tissue lysates was collected and mixed with 5x Protein Loading Buffer. Equal amount of total protein for each sample was loaded and run on a $4 \% \sim 20 \%$ SDS-PAGE gel. Samples were transferred to $0.45 \mu \mathrm{m}$ nitrocellulose (Invitrogen) and Western blotted with the antibodies listed in Supplemental Table 2, followed by the reaction with ECL substrates. The chemiluminescent signal was then detected by imaging the blot with Azure c600 imaging system. Bands were quantified using ImageJ software.

\section{Preparation of single cell suspension from mouse small intestine}

For single-cell RNA sequencing, mouse distal jejunums were collected $18.5 \mathrm{~h}$ after small intestinal ischemia/reperfusion surgery. Two mice were used for each group: Control I/R18.5h and EC-Foxc-DKO I/R-18.5h. Briefly, mice were anesthetized by isoflurane. Blood was removed by cardiac perfusion with cold PBS. The distal jejunum was then dissected from each individual, washed with cold PBS and cut into small pieces. The tissue was 
processed for scRNA-seq upon dissociating into single cell suspension in a digestion buffer (2 mg/mL Collagenase D, 0.2mg/mL DNAase I, $2 \mathrm{mg} / \mathrm{mL}$ Dispase II, 100 units $/ \mathrm{mL}$ penicillin and $100 \mu \mathrm{g} / \mathrm{mL}$ streptomycin in HBSS) (73) for $35 \mathrm{~min}$ at $37^{\circ} \mathrm{C}$, followed by filtration through a $70 \mu \mathrm{m}$ and a $40 \mu \mathrm{m}$ cell strainer. Cells were washed with washing buffer (0.5\% BSA, 2mM EDTA in PBS, $\mathrm{pH} 7.4)$ for three times, and resuspended in PBS with $0.04 \%$ BSA at a concentration of 1200 cells $/ \mu \mathrm{L}$ (according to 10x Genomics Document \#CG00053 Rev B) before being passed through a $30 \mu \mathrm{m}$ MACS SmartStrainer. The cell viability was tested by using the Cellometer Auto 2000 Cell Viability Counter (Nexcelom Bioscience, USA). The cell sample was processed for scRNA-seq only when the cell viability was more than $70 \%$. Average cell viability for samples was determined to be $80.96 \%$.

\section{Single cell 3' gene expression library construction and sequencing}

Single cell 3' gene expression libraries were constructed by using the Chromium Next GEM Single Cell 3' Reagent Kits v3.1 (10x Genomics, Pleasanton, CA, USA) according to the manufacturer's manual CG000204 Rev D. The single cell libraries were assessed for quality (TapeStation 4200, Agilent, Santa Clara, CA, USA) and then run by using pairedend $50 \mathrm{bp}$ sequencing on the Illumina HiSeq 4000 platform (Illumina, San Diego, CA, USA). 10,000 cells were targeted for each sample with a sequencing depth of 20,000 read pairs per cell.

\section{Pre-processing of single-cell RNA data}


Following library generation and sequencing, raw sequencing data were de-multiplexed and mapped to the mouse reference genome (mm10) using the CellRanger toolkit (10X Genomics, version 2.1.0). Gene expression matrices were then generated from both Control and EC-Foxc-DKO mice using CellRanger. The matrix files were then utilized for data processing and downstream analysis using the BIOMEX browser-based software platform and its incorporated packages developed in R (74). Quality control and data pretreatment was performed in BIOMEX with the following manually set parameters: i) genes with a row average of $<.001$ were excluded for downstream analysis and ii) cells in which over $10 \%$ of unique molecular identifiers (UMIs) were derived from the mitochondrial genome were considered as dead cells and removed from downstream analysis. The data were then normalized in BIOMEX using similar methodology to the NormalizeData function as implemented in the Seurat package (75).

\section{Variable gene identification, dimensionality reduction, clustering analysis, and differential gene expression analysis}

Following data pretreatment, BIOMEX was utilized for downstream dimensionality reduction of data and clustering analysis using the incorporated R packages. First, highly variable genes were identified utilizing the following feature selections: mean lower threshold $=0.01$, mean higher threshold $=8$, dispersion threshold $=0.5$. Data (using highly variable genes only) was then auto-scaled and summarized by principal component analysis (PCA), followed by visualization using Uniform Manifold Approximation and Projection (UMAP; top 15 principal components (PCs)) to reduce the data into a twodimensional space. Graph-based clustering was then performed in BIOMEX to cluster cells 
according to their respective gene expression profile using methodology similar to the FindClusters function in Seurat (clustering resolution $=0.5$, k-nearest neighbors $=15$ ). Marker set analysis was then performed in BIOMEX on highly variable genes to identify the top 10 gene markers expressed in each initial cluster using similar methodology described previously (76). Marker genes were then compared with single cell RNA-seq data from the small intestine of adult mice available from the Mouse Cell Atlas (MCA, http://bis.zju.edu.cn/MCA) and previously reported data (77) to identify transcriptionally unique cell populations. Clusters with highly similar expression patterns indicative of the same cell phenotype were merged into the same cluster. Differential gene expression analysis between Control and EC-Foxc-DKO mice for individual cell clusters was performed in BIOMEX using the Model-based Analysis of Single-cell Transcriptomics (MAST) package (78). $P$-values $<.05$ were considered statistically significant for differentially expressed genes.

\section{Data visualization}

BIOMEX implementation of the Plotly software was used for UMAP and volcano plot visualization.

Forkhead box $\mathrm{C}$ transcription factor binding prediction analysis in $\mathrm{RSPO}$ and

\section{CXCL12 loci}

Putative FOX-binding sites in the RSPO3 and CXCL12 loci were first identified using the Hypergeometric Optimization of Motif EnRichment (HOMER) (58) suite of tools to scan the entire Genome Reference Consortium Human Build 37 (GRCh37 or hg19) genome 
corresponding to the conserved RYMAAYA FOX transcription factor binding motif or the reported RYACACA FOXC transcription factor binding motif (79). The output files were then uploaded to the UCSC genome browser (57) to identify putative binding sites corresponding to transcriptionally active areas denoted by histone modification, DNAse sensitivity, and additional transcription factor chromatin immunoprecipitation data as per work reported and summarized on the Encyclopedia of DNA Elements (ENCODE; https://genome.ucsc.edu/ENCODE/)(2012). Putative sites in the human genome were then searched against the Genome Reference Consortium Mouse Build 38 (mm10) genome using the Evolutionary Conserved Region (ECR) Browser (https://ecrbrowser.dcode.org) and rVista 2.0 tools to identify conserved and aligned putative binding sites between mouse and human sequences. Conserved and aligned putative FOX binding site sequences are underlined and bolded within the human RSPO3 and CXCL12 ECRs shown below:

RSPO3 ECR-1.>hg19 chr6:127441643-127442074

CTCCTGGAGCTCGCGAGGGGCAGGAAGCGATGCTGCTGCTCTGGGCTCTCGC ACCTCCGCGGCGTGCAGCCCAGGCCCAGCGGCCCCACCTGGTTTCCCAAGGG GATCCCTCTCCCAGCGTCCCGCCCGGGAAGCCTTTCGGGGCAGACGTCGGGG CAGCGCGGAGGACACAACACCGGCTTGTGTGAGATGGAAATTTAGTAATCGC TTCTCGGCCGCTGTTTGTCCCTGCCCTGCCCCTGGGCATGAGGGTCCCCTGA AAGCTCCCTTTGTATTGCTGGCCTCGGCCAGACAAGGTTTGATTCACCTGAAA ATTGCCTTTTAAAAAGGTGGCAGTGCCAGAGCGACAGGGGCCCTGGCCGGGC CTCACCGCCTTTAGGATCACCTAGCAGGGGGCAGGAGAGCCCAAACTAAATA 
TGTCAGGGCATCCGATTTATTATTCTTGGCCTCCGAATGGTGTGTACTCTGGT AACTCCCCACCCACTCTTTCATTCCGGCCCGGACGCGGAAGGGGAGGCCTGG GGTTCAGCGGTGTCCACGCGCCTGGCGGTCCCCGCCTCCTCTCCTCCCAGGGT CTGGCCTGGGGAATTGAGTCTCAGGGTCAGTGGGCATAGGGAGCCCGGGCTG GAGCG

RSPO3 ECR-2.>hg19 chr6:127468065-127468506

TCTTGAAATGGATAATGATAGATTCCAAACACATGGAAATCTCTTGCCCCTTT TACTTTTTAGGATCTTTGCAAGCTTACAATATGTACACGTTTTCTGTAAGTCAC CAATGCTGAGTTACTGGCATGAAAAATGACCCTGTTACTTGGAAAGTAGTTTC ACTTACAAGTCCCCCAGGCCCTGTAATGTCTAAACCTCCTGTGCCACTTTATG TGACTACCCCGCCCCCACAGAGGAGCATGCACAGGAAAAGCAGACTTCCCTT

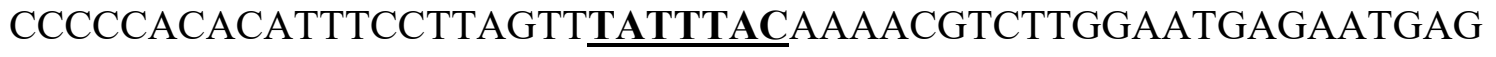
CTGCTTGTGGTTCCTGTGGCTGATTCAGGGATGGTTTCCTACAGGCAGAGGAT GCTGGTCAACCGAATGACCTCTCTGTAACTAACCCGTGCACCCCTGTGGTAA GGCTGTTTGGTCTTATAGGTACCTCTTCTAACTAAGCTTGGAGGGATTTGTTTT TGTGGTAAAGAACTTAGTAATAACCAAACGTCACTGTAAAGACAGATTTAAT AATGTTAAGGTCCATCAGAGCCTACTCCTTCTACTACCAACAAGAGAAGCCA GAAATACACTGGGATGCCTTTAGATTCCTGTGCATCAATCTTTCTTTCTCTAA GGATTATGG

RSPO3 ECR-3.>hg19 chr6:127497100-127497633 
AATACCAGTAAATGGGGACACATTATATTGAATAAGGGTATTGTTAGCCAAA TTCTAAGATTCATCTTAAATTGTTTTCTTATAAGAATTGTGTATTTACCATTTT AAAAATCACTATTATTTTAAAACACTTAGAAAGTGAACATTTGAAAATGATG TGCCTTTGGATGCTCTGTAATGTTAAGCAGATCCAGACATAAAGACAAAAGT AAATTCCAGAGTATTTTTGTAGCCATGGAATCACCATAAAAAGGGGTTTTTGA CCCCAATGTTACCGTAACATTGTCTTCAGCATTTCATATTTAATTACAGTAGA TTACTCACCAATATA CAAATGAAAACCACCCAAGAGGAGCCTCACCAAACCTGAGGTTGTCCAGATT GCATTGACTAAGATTAAGTAAAAGATCATTCATCTCCAGAGGTCATGCAATT AATCTCAGAGTGGGAGTTAAAGCAATGACTAAGCAGAAAAGGAAGCCAAAT ACAAGCTCGTAACAAAAGGTGCTGGGGCTCCAACATCAAGGAACTTGTTATT TCCTTTTTATTTATTTATTTTTTTTTAATAGACCTAAAACACTCATTCCTTACTA CTGGTTTCTTTGGGTCCTAAAATTCCACTTGGTTAGGTCAGCTATTTTCCATGA CTATTTTTGATACGGTCAAACAAATACAAAGAATAAGCTTTTAAAAAAC

RSPO3 ECR-4.>hg19 chr6:127498181-127498446

TTCACCTTATTGAGATTCAAAACCTTGTGTTTTAAACCAAGAATAATTTTGAA CTTGGTCGTTCCTTATTGCAAGGACTTCCTCCAACTAGGGTGAATTTTCACCC ACGATTAGCCTGCCTTTGGAGTTTATGATGTGAAGTGCCACAGCTGATGGCCC ATGAAAGAGACTGCTAAGTCTATTGCTCACATCAATATTCCCGCTGGATATTT TGCTCATTCCTTGGGAATTTCAAAGAATAACAAATA_ACCAACAAATCATCAA 
AAACTGTAACATGTTACCCAATTCTTGGGAATAAGCCAGTGAGACTAAAATC TGCTTAAGTATCACAAATGTGAATTCCCAGCATTTCAAGCTTGTAAACTGTAT CCAACACTCACCAATGTCTATTCCATTTCTTAGCTGTACAGAAGGGGCACATT CATCTGGTAACTGTCAACAATGAGGTTCCTTCCTTCTTGAACCC

CXCL12 ECR-1.>hg19 chr10:44888442-44889025

ATCAAAACCTTCACCTTTCTCTGCTGAAGGAATGGCCTTCTCTTATGGGCAGG GAGGGTTTCCTAGGGAAAGCCCACCCAGGCAGGAGATGAGGAGAGCAGCAT CTGAGCACACTTCATCCCACAGTGCCCATCCCATGAGTATCCTCCATAAATTA CAAAGAAAGAAAAAAAATAGGGAAAAAACAAACCTTTATTTCTCTGTTTAC TTCTCTGCATTTAATGAGCAGTTGTTAACATGACTGAAACCATCTGATGATTT TTACCAAATGGAAAAATCTGCCTACAGGGGCAATAAA_ATAAATATTCAGAAT AGAGAGAGGCAGTCATAAAAGACATTACCCGGTTGTAAACGGAGGCGGGTG GTGGTGATCTATTACCCCTGCCTCGGCAGCTTTCAACAGAGTTCTGGAATTCC AGGAGGGGCCCTGACCCAAGGCAA TATTTACTTCTGCGGCTTCTTCATCAGG TCAGCATGGGTATAATTCTGTCTACCAGTTGACTGGAGCTGAGGTTTCGAGCA GGAAGTGCAAACCCTGAGTGCTTATAACTCAGGAGGAGTGAGGCACCCCTTC CCAGAGTATGCCAAGAAAAGCACATTGTACTGTCCTGGCTGCAGGGGTGAGG CCCCTGCACACCCAGGCCATTATCAGCTTTGTGCCCTGGCCAACAGCGCCTCT GGTTGGTGCATTTGTCAGCTGTATTTTACCCTAGAGCTCTGGGAGGCTCATCC TTTTTTGGTATACCACCACGTGGAGAGAGCAGAGTTTTAATAGTGTGGCTGC 
CXCL12 ECR-2.>hg19 chr10:44872457-44872993

GGGTTAAAAAAAAAAAAAAAAGATCCAAAAACTTGAGCTGCAGATCTAATC TGCTCGTGAGAAAAGCCCATACACTGTCACACATGGGCTGTGAGAAGGGGTC TCAGACACCTGACTGCAGGCAGGCTTAACTATATAAACCAGAAACGTCTATA AGCTCCATCACTAACAACTAATGAATTTTATTTCAG TACAGTAGGACGTTTATACCATGAAACAATTAGCATTTTATTGCTAGTGCATA TAATGTCACATTTGATACAATTTTAGTACAAGTGAAAAAATACACTGTGGCTA ACATTGAAAAGCTGCAATCACATTTATATATCATATATATTTCTTTACAAATT GCCAGTAGTTTGAGATAATAGAGAAGTATAAACTACTGACATTCATATGGCT

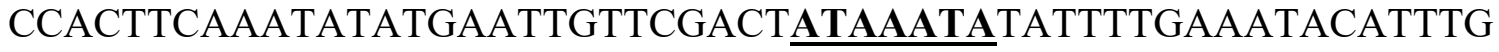
TTTTCTAAAGAAACGTAAAAAAAAATGTGCACAAAAATATATATAAAAAAAT GCCTTGCAAAAAGTTACAAATACCACCAGGACCTTCTGTGGATCGCATTTATG CATGGAAATGTCACCTTGCCAACAGTTCTGATTGGAACCTGAAACCCTGCTGT GGCTTCAGGAGGGGGTAGTGGCAAGATGATGGTTTATTCACTGATTTTTTCGC TTCTGATTTCGGAAACCTCAGAGTTTGTTAGTGCCTC CATGGCATACATAGGCT

CXCL12 ECR-3.>hg19 chr10:44871130-44871571

GCGCAGGCCTTCTAAGAGAGGAAGTGGAGGGCGGGCTGGGGGGCTCAGCAA CCTGGGCATTCCTGGAGCTCCCAGGCTATTCTGGACAGAGTCCTGAGCACAC AGGCTCCGCGTCACAGACCCCCGCCCCAGTCTCTGCACAACTACTTTCTTCAT 
AACTTAGCAGACCAACAAGGGCCTTAAAAAGCACATAAATACAGAAGCCGG TGTGGCTGGCAGGGCAGCAAAGCACTGCTCCCCCACGGAAGTCTGAGCCCCT GCCCAGTCTGCATGGGGGTCTGTCCTGGAGGAGGATCGAGCAAATTTACAAA GCGCCGAGAGCAAGTGAACTGTGGTCCATCTCGAGGTGGCAGATAACTAGTT TTTCCTTTTCTGGGCAGCCTTTCTCTTCTTCTGTCGCTTCTTTTTTCCTATCTTTT CTTTTTTCCCCACTTTTTCTTCTCTGCGCCCCCTTAGATAAAATTAGTAGAACC ATTAATAATGTGGAAATAAACAAAAGTTCGTCTCAGTCTGCATAAGATTTAA CACTGGCCCGTGTACTGGTTAAACTGTGCCTTCAGTCTCAGGCCTGGAGGCCC CTGGTCAAAGCACTGTTTATCAGTAATTCATTAAAATAAATTGGACATTTCCT TCCATTCAAAC

CXCL12 ECR-4.>hg19 chr10:44868531-44868889

GCCCAGTCTCCCCACCTGCACAGCTCAGAGAATACAAAACCCAGGAGCCCTG AGTCAGAGGAGTGGCTCCGTGGAAAGACACTCTAATAAGGCAAGTACAATA ATGGCCTTAGTCTAAGCTGCTACGTGTCGCCAGTGACACTGAATAATCAAACT AAATGAAAAATAAATGTCATGTATTTTGCTACATAATCAAATGGTGAAAATA TGGCAAAGTGTGCAAAACAAAGCCCTTGGCAATGCCTGGGGCCCTGCCCTGG CAGGGGAGGCTGTGGCAGGCCCTTCCCTAACACTGGTTTCAGAGCTGGGCTC CTACTGTAAGGGTTCCTCAGGCGTCTGACCCTCTCACATCTTGAACCTCC $\underline{\mathbf{T G T}}$ TGACAAGAACAGAGGGAAACACCATTAAGGCAGGTTCCCCCCACACCCAGC CAGCTCCAGAAGGACAGCGGCCTGCGCGGAGGCCCTGGGGCCAATCCCTACT 
TCCAAGTTGCCACCTTGGCCATGCATCTCCCTTCAGAGGCCCTGCGACAACAG ACGGGAGGGGAGTGCGAGA CGCAGCCAGGCTGAAGCGG

CXCL12 ECR-5.>hg19 chr10:44866547-44866769

GCAGCACCAGGTCCCGGAGGGACAGATGAGGGATCCCTAGTAAACAGCTCG TGGACGCACTTGACTAGCAGTTCGAAAGCAAAAAGAGATTCGGATTACAAGA GACTTTTCCCTTGCAAATGGAAGACTGTATTTAAATGCATATTGCTTTAGCTG GGCAGTCTCCAGGAAGGAGCTGATTGTTAGTAGAGGAATTGTTATGCAAATA ATTTTCCCTGCAGTTTCTACTCTTATCTATTTTCTCCCATGGAGGAGAAATAGA AATGATTCCTTTCCTGACTGTAGTCAAGATTTAGGGTCATGTTTGTTTCCTCA ACCAAGAATTCAATGGTCTTGGTTCATGGATTGGGGGCAGGTACATCCAAGT TCTACGTGACAGATTTTAAAATATCTTGGATTACCTGGATAATGACTGCCCCA CTG

\section{Mouse NEC model}

24-hour-old mice were submitted to a well characterized NEC model (40) conducted in a $33-35^{\circ} \mathrm{C}$ infant incubator or left with the dam. The NEC protocol includes: 1) initial orogastric inoculation with a standardized adult mouse commensal bacteria preparation ( $10^{8}$ colony-forming units) and LPS $(5 \mathrm{mg} / \mathrm{kg})$ to perturb the normal intestinal colonization process; 2) gavage with formula every $3 \mathrm{~h}$ (Esbilac, $200 \mathrm{ml} \cdot \mathrm{kg}-1 \cdot \mathrm{day}-1$ ); and 3 ) exposure to brief episodes of hypoxia ( $60 \mathrm{~s}$ in $100 \% \mathrm{~N} 2$ ) followed immediately by cold stress (10 min at $4^{\circ} \mathrm{C}$ ) twice daily. This protocol induces intestinal injuries ranging from epithelial 
injury to transmural necrosis resembling human NEC which typically develop after 36 hours and has been widely used to study NEC pathogenesis ( 80$)$. Pups were euthanized by decapitation at 24 hours into NEC and whole intestinal tissues were collected and fixed in formalin for 24 hours before tissue processing and block preparation.

\section{Quantification}

For quantification of BrdU+ or CCND1+ cells in crypts, images from different fields of section under a 20x objective were acquired and at least 50 crypts were analyzed for each sample. The number of BrdU+ or CCND1+ cells was counted for each crypt. For analysis of BEC and LEC proliferation and apoptosis, confocal Z-stacks were acquired using a 20x objective from at least 8 different fields of $15 \mu \mathrm{m}$ paraffin sections for each sample. Area of blood vessels (CD31+LYVE1-) and lymphatic vessels (CD31+LYVE1+) were measured using ImageJ software. BrdU+ or TUNEL+ cells in blood and lymphatic vessels were counted, then the number of BrdU+ or TUNEL+ cells per $0.1 \mathrm{~mm}^{2}$ vessel areas was calculated and compared between groups. Measurements for the length of blood capillaries and lacteals was performed as previously described (71). The numbers of branches and branching points for the villous blood vasculature was calculated based on the wholemount staining of VEGFR2 as previously described (71) using ImageJ software. Around $30 \sim 50$ villi were analyzed for each sample. For CD31+ vessel density quantification, 8 10 images were acquired using a 20x objective and the CD31+ vessel area and the intestinal tissue area in the images were measured using ImageJ software. CD31+ vessel density percentage was determined as the total CD31+ vessel area/total intestinal tissue area $\mathrm{x}$ $100 \%$. 


\section{Statistics}

For quantification, statistical analysis was performed using GraphPad Prism 8.0 (GraphPad Software). $P$ values were obtained by performing Mann-Whitney $U$ test or Kruskal-Wallis One-way ANOVA test. Data are presented as mean \pm SEM (standard error of the mean) of representative experiments from at least three biological replicates. $P$ values $<0.05$ were considered statistically significant. For scRNA-seq data, differential gene expression analysis between groups for individual cell clusters was performed in BIOMEX using the Model-based Analysis of Single-cell Transcriptomics (MAST) package (78). Adjusted $P$ values $<0.05$ were considered statistically significant for differentially expressed genes.

\section{Study approval}

All experimental protocols and procedures used in this study were approved by the Institutional Animal Care and Use Committee (IACUC) at Northwestern University.

\section{References}

1. D. J. Nolan, M. Ginsberg, E. Israely, B. Palikuqi, M. G. Poulos, D. James, B. S. Ding, W. Schachterle, Y. Liu, Z. Rosenwaks, J. M. Butler, J. Xiang, A. Rafii, K. Shido, S. Y. Rabbany, O. Elemento, S. Rafii, Molecular signatures of tissue-specific microvascular endothelial cell heterogeneity in organ maintenance and regeneration. Developmental cell 26, 204-219 (2013).

2. S. Rafii, J. M. Butler, B. S. Ding, Angiocrine functions of organ-specific endothelial cells. Nature 529, 316-325 (2016). 
3. S. Bertoni, V. Ballabeni, E. Barocelli, M. Tognolini, Mesenteric ischemia-reperfusion: an overview of preclinical drug strategies. Drug Discov Today 23, 1416-1425 (2018).

4. L. M. Gonzalez, A. J. Moeser, A. T. Blikslager, Animal models of ischemia-reperfusioninduced intestinal injury: progress and promise for translational research. Am J Physiol Gastrointest Liver Physiol 308, G63-75 (2015).

5. R. M. Bowker, X. Yan, I. G. De Plaen, Intestinal microcirculation and necrotizing enterocolitis: The vascular endothelial growth factor system. Semin Fetal Neonatal Med 23, 411-415 (2018).

6. R. D. Shelby, R. Raab, G. E. Besner, S. J. McElroy, Hope on the horizon: promising novel therapies for necrotizing enterocolitis. Pediatr Res 88, 30-34 (2020).

7. N. Barker, J. H. van Es, J. Kuipers, P. Kujala, M. van den Born, M. Cozijnsen, A. Haegebarth, J. Korving, H. Begthel, P. J. Peters, H. Clevers, Identification of stem cells in small intestine and colon by marker gene Lgr5. Nature 449, 1003-1007 (2007).

8. K. S. Yan, L. A. Chia, X. Li, A. Ootani, J. Su, J. Y. Lee, N. Su, Y. Luo, S. C. Heilshorn, M. R. Amieva, E. Sangiorgi, M. R. Capecchi, C. J. Kuo, The intestinal stem cell markers Bmil and Lgr5 identify two functionally distinct populations. Proceedings of the National Academy of Sciences of the United States of America 109, 466-471 (2012).

9. C. Metcalfe, N. M. Kljavin, R. Ybarra, F. J. de Sauvage, Lgr5+ stem cells are indispensable for radiation-induced intestinal regeneration. Cell stem cell 14, 149-159 (2014).

10. L. M. Gonzalez, A. S. Stewart, J. Freund, C. R. Kucera, C. M. Dekaney, S. T. Magness, A. T. Blikslager, Preservation of reserve intestinal epithelial stem cells following severe ischemic injury. Am J Physiol Gastrointest Liver Physiol 316, G482-G494 (2019). 
11. C. A. Richmond, H. Rickner, M. S. Shah, T. Ediger, L. Deary, F. Zhou, A. Tovaglieri, D. L. Carlone, D. T. Breault, JAK/STAT-1 Signaling Is Required for Reserve Intestinal Stem Cell Activation during Intestinal Regeneration Following Acute Inflammation. Stem Cell Reports 10, 17-26 (2018).

12. A. J. M. Santos, Y. H. Lo, A. T. Mah, C. J. Kuo, The Intestinal Stem Cell Niche: Homeostasis and Adaptations. Trends Cell Biol 28, 1062-1078 (2018).

13. Z. Steinhart, S. Angers, Wnt signaling in development and tissue homeostasis. Development 145, (2018).

14. R. Aoki, M. Shoshkes-Carmel, N. Gao, S. Shin, C. L. May, M. L. Golson, A. M. Zahm, M. Ray, C. L. Wiser, C. V. Wright, K. H. Kaestner, Foxl1-expressing mesenchymal cells constitute the intestinal stem cell niche. Cell Mol Gastroenterol Hepatol 2, 175-188 (2016).

15. N. McCarthy, J. Kraiczy, R. A. Shivdasani, Cellular and molecular architecture of the intestinal stem cell niche. Nature cell biology 22, 1033-1041 (2020).

16. N. McCarthy, E. Manieri, E. E. Storm, A. Saadatpour, A. M. Luoma, V. N. Kapoor, S. Madha, L. T. Gaynor, C. Cox, S. Keerthivasan, K. Wucherpfennig, G. C. Yuan, F. J. de Sauvage, S. J. Turley, R. A. Shivdasani, Distinct Mesenchymal Cell Populations Generate the Essential Intestinal BMP Signaling Gradient. Cell stem cell 26, 391-402 e395 (2020).

17. H. F. Farin, J. H. Van Es, H. Clevers, Redundant sources of Wnt regulate intestinal stem cells and promote formation of Paneth cells. Gastroenterology 143, 1518-1529 e1517 (2012). 
18. M. Shoshkes-Carmel, Y. J. Wang, K. J. Wangensteen, B. Toth, A. Kondo, E. E. Massasa, S. Itzkovitz, K. H. Kaestner, Subepithelial telocytes are an important source of Wnts that supports intestinal crypts. Nature 557, 242-246 (2018).

19. T. Valenta, B. Degirmenci, A. E. Moor, P. Herr, D. Zimmerli, M. B. Moor, G. Hausmann, C. Cantu, M. Aguet, K. Basler, Wnt Ligands Secreted by Subepithelial Mesenchymal Cells Are Essential for the Survival of Intestinal Stem Cells and Gut Homeostasis. Cell reports 15, 911-918 (2016).

20. E. E. Storm, S. Durinck, F. de Sousa e Melo, J. Tremayne, N. Kljavin, C. Tan, X. Ye, C. Chiu, T. Pham, J. A. Hongo, T. Bainbridge, R. Firestein, E. Blackwood, C. Metcalfe, E. W. Stawiski, R. L. Yauch, Y. Wu, F. J. de Sauvage, Targeting PTPRK-RSPO3 colon tumours promotes differentiation and loss of stem-cell function. Nature 529, 97-100 (2016).

21. L. Kannan, K. Kis-Toth, K. Yoshiya, T. H. Thai, S. Sehrawat, T. N. Mayadas, J. J. Dalle Lucca, G. C. Tsokos, R-spondin3 prevents mesenteric ischemia/reperfusion-induced tissue damage by tightening endothelium and preventing vascular leakage. Proceedings of the National Academy of Sciences of the United States of America 110, 14348-14353 (2013).

22. R. Ogasawara, D. Hashimoto, S. Kimura, E. Hayase, T. Ara, S. Takahashi, H. Ohigashi, K. Yoshioka, T. Tateno, E. Yokoyama, K. Ebata, T. Kondo, J. Sugita, M. Onozawa, T. Iwanaga, T. Teshima, Intestinal Lymphatic Endothelial Cells Produce R-Spondin3. Scientific reports 8, 10719 (2018). 
23. E. M. García-Cuesta, C. A. Santiago, J. Vallejo-Díaz, Y. Juarranz, J. M. RodríguezFrade, M. Mellado, The Role of the CXCL12/CXCR4/ACKR3 Axis in Autoimmune Diseases. Front Endocrinol (Lausanne) 10, 585 (2019).

24. S. P. Herbert, D. Y. Stainier, Molecular control of endothelial cell behaviour during blood vessel morphogenesis. Nat Rev Mol Cell Biol 12, 551-564 (2011).

25. C. Hitchon, K. Wong, G. Ma, J. Reed, D. Lyttle, H. El-Gabalawy, Hypoxia-induced production of stromal cell-derived factor 1 (CXCL12) and vascular endothelial growth factor by synovial fibroblasts. Arthritis Rheum 46, 2587-2597 (2002).

26. B. Santiago, E. Calonge, M. J. Del Rey, I. Gutierrez-Canas, E. Izquierdo, A. Usategui, M. Galindo, J. Alcami, J. L. Pablos, CXCL12 gene expression is upregulated by hypoxia and growth arrest but not by inflammatory cytokines in rheumatoid synovial fibroblasts.

Cytokine 53, 184-190 (2011).

27. I. Kryczek, A. Lange, P. Mottram, X. Alvarez, P. Cheng, M. Hogan, L. Moons, S. Wei, L. Zou, V. Machelon, D. Emilie, M. Terrassa, A. Lackner, T. J. Curiel, P. Carmeliet, W. Zou, CXCL12 and vascular endothelial growth factor synergistically induce neoangiogenesis in human ovarian cancers. Cancer research 65, 465-472 (2005).

28. K. Yamada, N. Maishi, K. Akiyama, M. Towfik Alam, N. Ohga, T. Kawamoto, M. Shindoh, N. Takahashi, T. Kamiyama, Y. Hida, A. Taketomi, K. Hida, CXCL12-CXCR7 axis is important for tumor endothelial cell angiogenic property. Int $J$ Cancer 137, 2825$2836(2015)$.

29. P. Chang, B. Zhang, L. Shao, W. Song, W. Shi, L. Wang, T. Xu, D. Li, X. Gao, Y. Qu, L. Dong, J. Wang, Mesenchymal stem cells over-expressing cxcl12 enhance the radioresistance of the small intestine. Cell death \& disease 9, 154 (2018). 
30. N. P. Zimmerman, R. A. Vongsa, S. L. Faherty, N. H. Salzman, M. B. Dwinell, Targeted intestinal epithelial deletion of the chemokine receptor CXCR4 reveals important roles for extracellular-regulated kinase-1/2 in restitution. Lab Invest 91, 1040-1055 (2011).

31. T. Kume, The Cooperative Roles of Foxc1 and Foxc2 in Cardiovascular Development. Forkhead Transcription Factors: Vital Elements in Biology and Medicine 665, 63-77 (2009).

32. E. W. Lam, J. J. Brosens, A. R. Gomes, C. Y. Koo, Forkhead box proteins: tuning forks for transcriptional harmony. Nature reviews. Cancer 13, 482-495 (2013).

33. M. Seifi, M. A. Walter, Axenfeld-Rieger syndrome. Clinical genetics 93, 1123-1130 (2018).

34. J. Fang, S. L. Dagenais, R. P. Erickson, M. F. Arlt, M. W. Glynn, J. L. Gorski, L. H. Seaver, T. W. Glover, Mutations in FOXC2 (MFH-1), a forkhead family transcription factor, are responsible for the hereditary lymphedema-distichiasis syndrome. American journal of human genetics 67, 1382-1388 (2000).

35. F. Becker, S. Potepalov, R. Shehzahdi, M. Bernas, M. Witte, F. Abreo, J. Traylor, W. A. Orr, I. Tsunoda, J. S. Alexander, Downregulation of FoxC2 Increased Susceptibility to Experimental Colitis: Influence of Lymphatic Drainage Function? Inflammatory bowel diseases 21, 1282-1296 (2015).

36. K. Yoshiya, P. H. Lapchak, T. H. Thai, L. Kannan, P. Rani, J. J. Dalle Lucca, G. C. Tsokos, Depletion of gut commensal bacteria attenuates intestinal ischemia/reperfusion injury. Am J Physiol Gastrointest Liver Physiol 301, G1020-1030 (2011).

37. C. Hader, A. Marlier, L. Cantley, Mesenchymal-epithelial transition in epithelial response to injury: the role of Foxc2. Oncogene 29, 1031-1040 (2010). 
38. M. B. Amin, N. Miura, M. K. Uddin, M. J. Islam, N. Yoshida, S. Iseki, T. Kume, P. A. Trainor, H. Saitsu, K. Aoto, Foxc2CreERT2 knock-in mice mark stage-specific Foxc2expressing cells during mouse organogenesis. Congenital anomalies 57, 24-31 (2017).

39. M. D. Muzumdar, B. Tasic, K. Miyamichi, L. Li, L. Luo, A global double-fluorescent Cre reporter mouse. Genesis 45, 593-605 (2007).

40. R. Tian, S. X. Liu, C. Williams, T. D. Soltau, R. Dimmitt, X. Zheng, I. G. De Plaen, Characterization of a necrotizing enterocolitis model in newborn mice. Int J Clin Exp Med 3, 293-302 (2010).

41. T. Kume, K. Deng, B. L. Hogan, Minimal phenotype of mice homozygous for a null mutation in the forkhead/winged helix gene, Mf2. Molecular and cellular biology 20, 1419-1425 (2000).

42. T. Kume, H. Jiang, J. M. Topczewska, B. L. Hogan, The murine winged helix transcription factors, Foxc1 and Foxc2, are both required for cardiovascular development and somitogenesis. Genes \& development 15, 2470-2482 (2001).

43. S. Seo, H. Fujita, A. Nakano, M. Kang, A. Duarte, T. Kume, The forkhead transcription factors, Foxc1 and Foxc2, are required for arterial specification and lymphatic sprouting during vascular development. Developmental biology 294, 458-470 (2006).

44. A. Sasman, C. Nassano-Miller, K. S. Shim, H. Y. Koo, T. Liu, K. M. Schultz, M. Millay, A. Nanano, M. Kang, T. Suzuki, T. Kume, Generation of conditional alleles for Foxc1 and Foxc2 in mice. Genesis 50, 766-774 (2012).

45. I. Sorensen, R. H. Adams, A. Gossler, DLL1-mediated Notch activation regulates endothelial identity in mouse fetal arteries. Blood 113, 5680-5688 (2009). 
46. P. R. Norden, A. Sabine, Y. Wang, C. S. Demir, T. Liu, T. V. Petrova, T. Kume, Shear stimulation of FOXC1 and $\mathrm{FOXC} 2$ differentially regulates cytoskeletal activity during lymphatic valve maturation. eLife 9, (2020).

47. C. J. Chiu, A. H. McArdle, R. Brown, H. J. Scott, F. N. Gurd, Intestinal mucosal lesion in low-flow states. I. A morphological, hemodynamic, and metabolic reappraisal. Arch Surg 101, 478-483 (1970).

48. N. F. Cerqueira, C. A. Hussni, W. B. Yoshida, Pathophysiology of mesenteric ischemia/reperfusion: a review. Acta Cir Bras 20, 336-343 (2005).

49. L. G. van der Flier, A. Haegebarth, D. E. Stange, M. van de Wetering, H. Clevers, OLFM4 is a robust marker for stem cells in human intestine and marks a subset of colorectal cancer cells. Gastroenterology 137, 15-17 (2009).

50. I. Martinez-Corral, L. Stanczuk, M. Frye, M. H. Ulvmar, R. Dieguez-Hurtado, D. Olmeda, T. Makinen, S. Ortega, Vegfr3-CreER (T2) mouse, a new genetic tool for targeting the lymphatic system. Angiogenesis 19, 433-445 (2016).

51. F. W. Meng, H. Shimoda, T. Kajiwara, M. Matsuda, S. Kato, Reconstruction of central lacteals in the murine jejunum following ischemia-reperfusion injury. Archives of histology and cytology 70, 135-146 (2007).

52. N. McCarthy, E. Manieri, E. E. Storm, A. Saadatpour, A. M. Luoma, V. N. Kapoor, S. Madha, L. T. Gaynor, C. Cox, S. Keerthivasan, K. Wucherpfennig, G. C. Yuan, F. J. de Sauvage, S. J. Turley, R. A. Shivdasani, Distinct Mesenchymal Cell Populations Generate the Essential Intestinal BMP Signaling Gradient. Cell Stem Cell 26, 391402.e395 (2020). 
53. N. V. Lee, M. Sato, D. S. Annis, J. A. Loo, L. Wu, D. F. Mosher, M. L. Iruela-Arispe, ADAMTS1 mediates the release of antiangiogenic polypeptides from TSP1 and 2. The EMBO journal 25, 5270-5283 (2006).

54. M. Obika, H. Ogawa, K. Takahashi, J. Li, O. F. Hatipoglu, M. Z. Cilek, T. Miyoshi, J. Inagaki, T. Ohtsuki, S. Kusachi, Y. Ninomiya, S. Hirohata, Tumor growth inhibitory effect of ADAMTS1 is accompanied by the inhibition of tumor angiogenesis. Cancer science 103, 1889-1897 (2012).

55. M. R. Garnica, L. V. de Moraes, L. V. Rizzo, H. F. de Andrade, Jr., Supplementation of CXCL12 (CXCL12) induces homing of CD11c+ dendritic cells to the spleen and enhances control of Plasmodium berghei malaria in BALB/c mice. Immunology 115, 399-406 (2005).

56. A. Gonzalez-Loyola, E. Bovay, J. Kim, T. W. Lozano, A. Sabine, F. Renevey, S. ArrozMadeira, A. Rapin, T. P. Wypych, G. Rota, S. Durot, D. Velin, B. Marsland, G. Guarda, M. Delorenzi, N. Zamboni, S. A. Luther, T. V. Petrova, FOXC2 controls adult lymphatic endothelial specialization, function, and gut lymphatic barrier preventing multiorgan failure. Sci $A d v$ 7, (2021).

57. W. J. Kent, C. W. Sugnet, T. S. Furey, K. M. Roskin, T. H. Pringle, A. M. Zahler, D. Haussler, The human genome browser at UCSC. Genome research 12, 996-1006 (2002).

58. S. Heinz, C. Benner, N. Spann, E. Bertolino, Y. C. Lin, P. Laslo, J. X. Cheng, C. Murre, H. Singh, C. K. Glass, Simple combinations of lineage-determining transcription factors prime cis-regulatory elements required for macrophage and B cell identities. Mol Cell 38, 576-589 (2010). 
59. S. Liu, Y. Yin, R. Yu, Y. Li, W. Zhang, R-spondin3-LGR4 signaling protects hepatocytes against DMOG-induced hypoxia/reoxygenation injury through activating beta-catenin. Biochemical and biophysical research communications 499, 59-65 (2018).

60. B. Zhou, L. Magana, Z. Hong, L. S. Huang, S. Chakraborty, Y. Tsukasaki, C. Huang, L. Wang, A. Di, B. Ganesh, X. Gao, J. Rehman, A. B. Malik, The angiocrine Rspondin3 instructs interstitial macrophage transition via metabolic-epigenetic reprogramming and resolves inflammatory injury. Nature immunology 21, 1430-1443 (2020).

61. E. Kostallari, V. H. Shah, Angiocrine signaling in the hepatic sinusoids in health and disease. Am J Physiol Gastrointest Liver Physiol 311, G246-251 (2016).

62. D. Ribatti, R. Tamma, T. Annese, The role of vascular niche and endothelial cells in organogenesis and regeneration. Experimental cell research 398, 112398 (2021).

63. D. Yi, B. Liu, T. Wang, Q. Liao, M. M. Zhu, Y. Y. Zhao, Z. Dai, Endothelial Autocrine Signaling through CXCL12/CXCR4/FoxM1 Axis Contributes to Severe Pulmonary Arterial Hypertension. Int J Mol Sci 22, (2021).

64. Y. Omatsu, M. Seike, T. Sugiyama, T. Kume, T. Nagasawa, Foxc1 is a critical regulator of haematopoietic stem/progenitor cell niche formation. Nature 508, 536-540 (2014).

65. W. Xia, J. Zhu, X. Wang, Y. Tang, P. Zhou, X. Wei, B. Chang, X. Zheng, W. Zhu, M. Hou, S. Li, Overexpression of Foxc1 regenerates crushed rat facial nerves by promoting Schwann cells migration via the Wnt/beta-catenin signaling pathway. Journal of cellular physiology 235, 9609-9622 (2020).

66. E. Kaufmann, W. Knochel, Five years on the wings of fork head. Mechanisms of development 57, 3-20. (1996). 
67. D. G. Overdier, A. Porcella, R. H. Costa, The DNA-binding specificity of the hepatocyte nuclear factor 3/forkhead domain is influenced by amino-acid residues adjacent to the recognition helix. Molecular and cellular biology 14, 2755-2766. (1994).

68. S. Pierrou, M. Hellqvist, L. Samuelsson, S. Enerback, P. Carlsson, Cloning and characterization of seven human forkhead proteins: binding site specificity and DNA bending. The EMBO journal 13, 5002-5012. (1994).

69. A. N. Stratman, M. J. Davis, G. E. Davis, VEGF and FGF prime vascular tube morphogenesis and sprouting directed by hematopoietic stem cell cytokines. Blood $\mathbf{1 1 7}$, 3709-3719 (2011).

70. K. Bersell, S. Choudhury, M. Mollova, B. D. Polizzotti, B. Ganapathy, S. Walsh, B. Wadugu, S. Arab, B. Kuhn, Moderate and high amounts of tamoxifen in alphaMHCMerCreMer mice induce a DNA damage response, leading to heart failure and death. Dis Model Mech 6, 1459-1469 (2013).

71. J. Bernier-Latmani, T. V. Petrova, High-resolution 3D analysis of mouse small-intestinal stroma. Nature protocols 11, 1617-1629 (2016).

72. T. K. Niethamer, C. T. Stabler, J. P. Leach, J. A. Zepp, M. P. Morley, A. Babu, S. Zhou, E. E. Morrisey, Defining the role of pulmonary endothelial cell heterogeneity in the response to acute lung injury. Elife $\mathbf{9}$, (2020).

73. N. Joshi, S. Watanabe, R. Verma, R. P. Jablonski, C. I. Chen, P. Cheresh, N. S. Markov, P. A. Reyfman, A. C. McQuattie-Pimentel, L. Sichizya, Z. Lu, R. Piseaux-Aillon, D. Kirchenbuechler, A. S. Flozak, C. J. Gottardi, C. M. Cuda, H. Perlman, M. Jain, D. W. Kamp, G. R. S. Budinger, A. V. Misharin, A spatially restricted fibrotic niche in 
pulmonary fibrosis is sustained by M-CSF/M-CSFR signalling in monocyte-derived alveolar macrophages. Eur Respir J 55, (2020).

74. F. Taverna, J. Goveia, T. K. Karakach, S. Khan, K. Rohlenova, L. Treps, A. Subramanian, L. Schoonjans, M. Dewerchin, G. Eelen, P. Carmeliet, BIOMEX: an interactive workflow for (single cell) omics data interpretation and visualization. Nucleic acids research 48, W385-W394 (2020).

75. R. Satija, J. A. Farrell, D. Gennert, A. F. Schier, A. Regev, Spatial reconstruction of single-cell gene expression data. Nat Biotechnol 33, 495-502 (2015).

76. J. Kalucka, L. de Rooij, J. Goveia, K. Rohlenova, S. J. Dumas, E. Meta, N. V. Conchinha, F. Taverna, L. A. Teuwen, K. Veys, M. García-Caballero, S. Khan, V. Geldhof, L. Sokol, R. Chen, L. Treps, M. Borri, P. de Zeeuw, C. Dubois, T. K. Karakach, K. D. Falkenberg, M. Parys, X. Yin, S. Vinckier, Y. Du, R. A. Fenton, L. Schoonjans, M. Dewerchin, G. Eelen, B. Thienpont, L. Lin, L. Bolund, X. Li, Y. Luo, P. Carmeliet, Single-Cell Transcriptome Atlas of Murine Endothelial Cells. Cell 180, 764-779.e720 (2020).

77. A. L. Haber, M. Biton, N. Rogel, R. H. Herbst, K. Shekhar, C. Smillie, G. Burgin, T. M. Delorey, M. R. Howitt, Y. Katz, I. Tirosh, S. Beyaz, D. Dionne, M. Zhang, R. Raychowdhury, W. S. Garrett, O. Rozenblatt-Rosen, H. N. Shi, O. Yilmaz, R. J. Xavier, A. Regev, A single-cell survey of the small intestinal epithelium. Nature 551, 333-339 (2017).

78. G. Finak, A. McDavid, M. Yajima, J. Deng, V. Gersuk, A. K. Shalek, C. K. Slichter, H. W. Miller, M. J. McElrath, M. Prlic, P. S. Linsley, R. Gottardo, MAST: a flexible 
statistical framework for assessing transcriptional changes and characterizing heterogeneity in single-cell RNA sequencing data. Genome biology 16, 278 (2015).

79. X. Chen, H. Wei, J. Li, X. Liang, S. Dai, L. Jiang, M. Guo, L. Qu, Z. Chen, L. Chen, Y. Chen, Structural basis for DNA recognition by FOXC2. Nucleic acids research 47, 37523764 (2019).

80. X. Yan, E. Managlia, S. X. Liu, X. D. Tan, X. Wang, C. Marek, I. G. De Plaen, Lack of VEGFR2 signaling causes maldevelopment of the intestinal microvasculature and facilitates necrotizing enterocolitis in neonatal mice. Am J Physiol Gastrointest Liver Physiol 310, G716-725 (2016).

\section{Acknowledgments}

We thank William Muller (Northwestern University) and Bona Jabri (University of Chicago) for helpful advice. Cdh5-Cre $e^{E R T 2}$ mice were kindly provided by Dr. Ralf Adams at the Max-Planck-Institute for Molecular Biomedicine, Germany. Single cell RNA-seq experiments were performed at the NUSeq Core Facility at Northwestern University. Funding: This work was supported by the NIH (R01HL126920 and R01HL144129 to TK). Imaging work was performed at the Northwestern University Center for Advanced Microscopy generously supported by NCI CCSG P30 CA060553 awarded to the Robert H Lurie Comprehensive Cancer Center. Author Contributions: CT, PRN, NU, XY, IGD, and TK designed and analyzed the experiments. CT, XY, TL, NU, and TL conducted the experiments. PRN analyzed scRNA-seq data. KA and SO provided Cre mouse lines. CT, PRN, and TK wrote the manuscript. Competing Interests: C. Tan, None; P.R. Norden, 
bioRxiv preprint doi: https://doi.org/10.1101/2022.03.03.482713; this version posted March 3, 2022. The copyright holder for this preprint (which was not certified by peer review) is the author/funder, who has granted bioRxiv a license to display the preprint in perpetuity. It is made available under aCC-BY-NC-ND 4.0 International license.

None; T. Liu, None; N. Ujiie, None; X. Yan, None; K Aoto, None; S. Ortega, None; I.G.

De Plaen, None; T. Kume, None. 


\section{Figures and Figure legends}

A

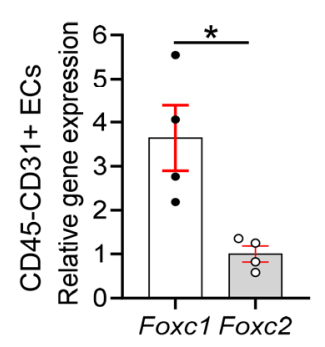

E

CD31/LYVE1
B

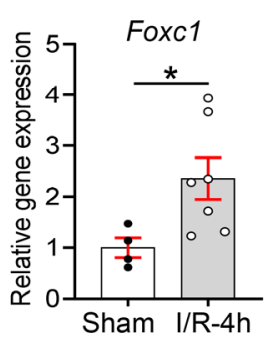

C

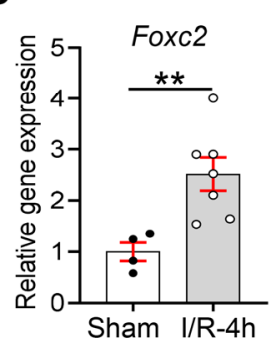

D

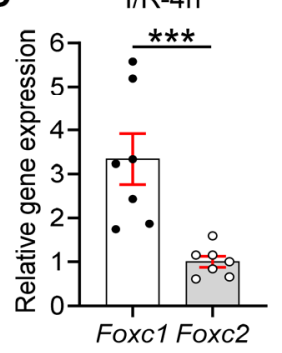

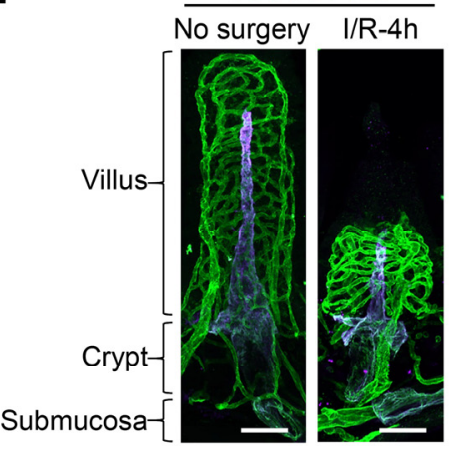
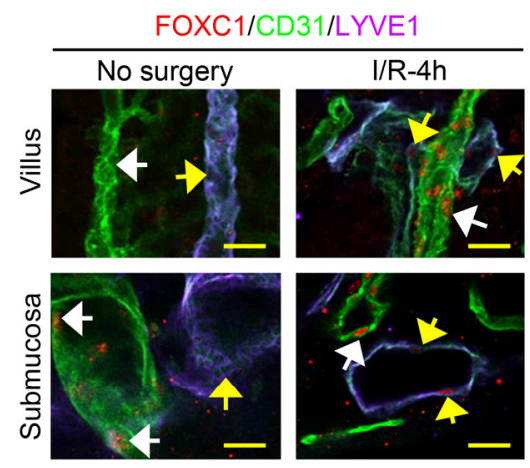

$\mathbf{F}$
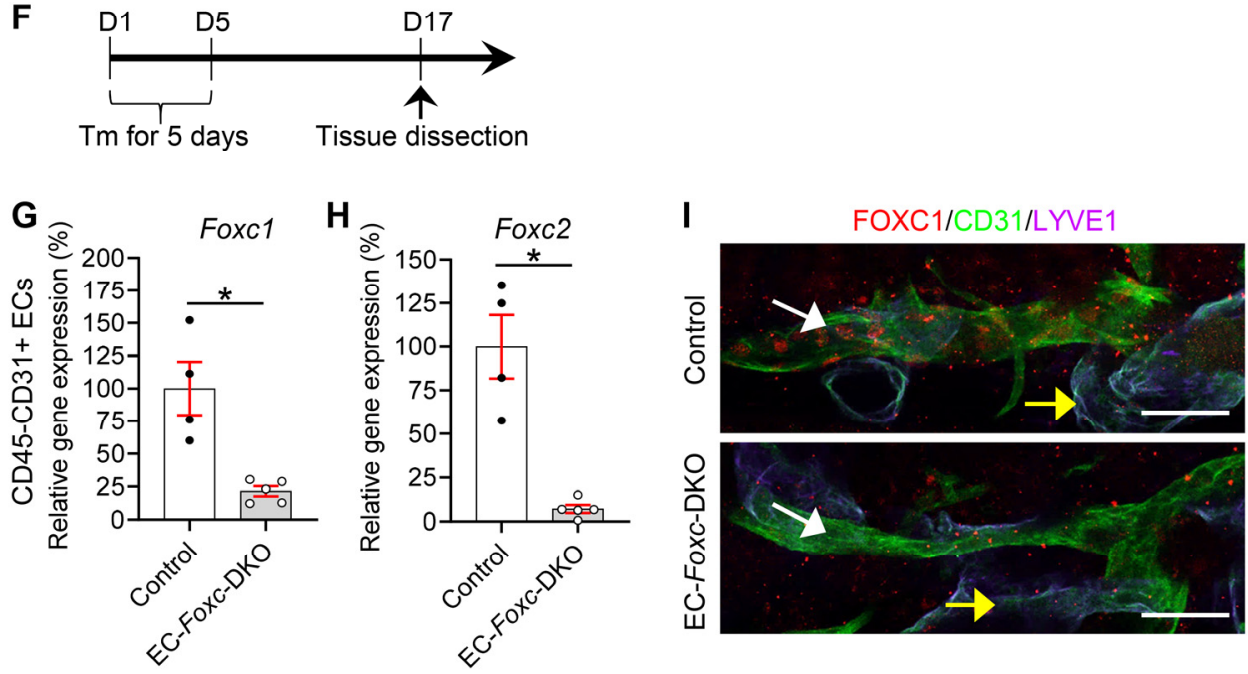

Fig. 1. Expression levels of Foxc1 and Foxc2 in the mouse small intestine. Relative expression level(s) of (A) Foxc1 and Foxc2 under no surgery, (B) Foxc1 and (C) Foxc2 in sham and I/R-4h intestines, (D) both Foxcl and Foxc2 at I/R-4h, in the isolated CD45CD31+ ECs from Foxc $1^{f l f l} ; F_{\text {oxc }} 2^{f l f l}$ mouse distal jejunums. Data are mean \pm SEM, MannWhitney $U$ test, $\mathrm{N}=4 \sim 7,{ }^{*} P<0.05, * * P<0.01, * * * P<0.001$. (E) Representative images of 
the whole-mount villi stained with FOXC1/CD31/LYVE1 in Foxc $1^{f l / f l} ; F_{\text {oxc }} 2^{f l f l}$ mice. Images of maximum intensity projections (left panel, CD31/LYVE1) show the blood and lymphatic vasculatures from villus to submucosa. Images of optical sections with high magnification (right panels, FOXC1/CD31/LYVE1) show the expression of FOXC1 in BECs/LECs (white/yellow arrows). White scale bars $=50 \mu \mathrm{m}$, yellow scale bars $=20 \mu \mathrm{m}$. (F) Schematic showing the time of Tamoxifen (Tm) injection and tissue dissection. Relative expression of Foxc1 (G) and Foxc2 (H) in isolated CD45-CD31+ ECs from distal jejunums $12 \mathrm{~d}$ after Tm treatment. Data are mean \pm SEM, Mann-Whitney $U$ test, $N=4 \sim 5$, $* P<0.05$. (I) Representative confocal images of submucosal blood vessels/lymphatics (white/yellow arrows) in whole-mount intestines stained with FOXC1/CD31/LYVE1. In control, FOXC1 is expressed in the nuclei of the BECs, but hardly detectable in LECs. FOXC1 is down-regulated in BECs after Tm treatment in EC-Foxc-DKO mice compared with control. Scale bars $=50 \mu \mathrm{m}$. Note that the red tiny spots outside the vasculatures are non-specific staining. 


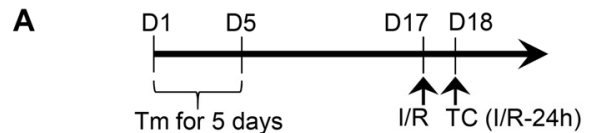

B
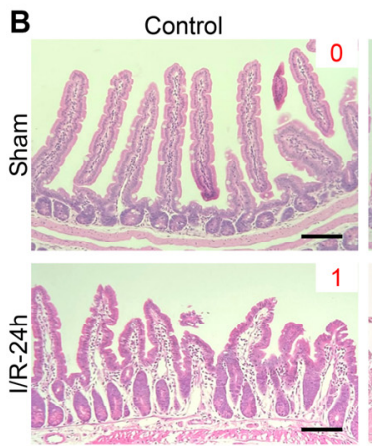

$\mathbf{F}$
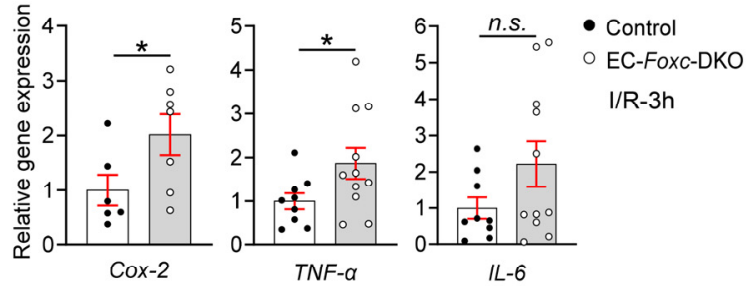

IL-6
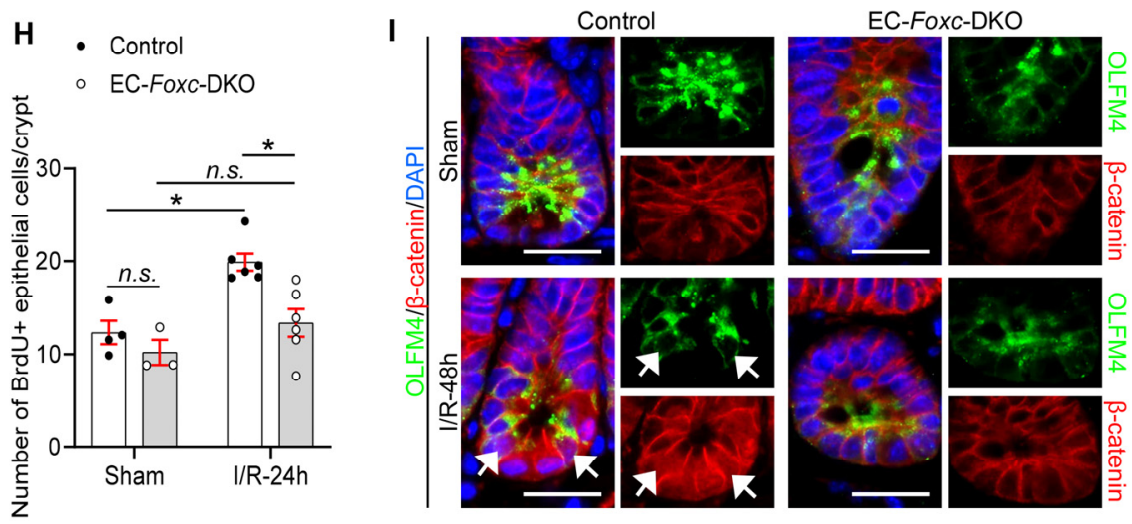

D

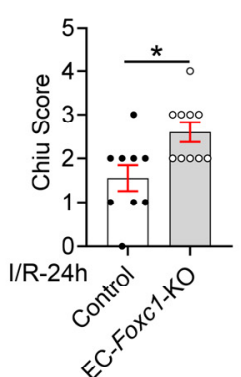

E

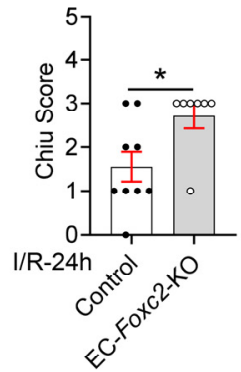

G
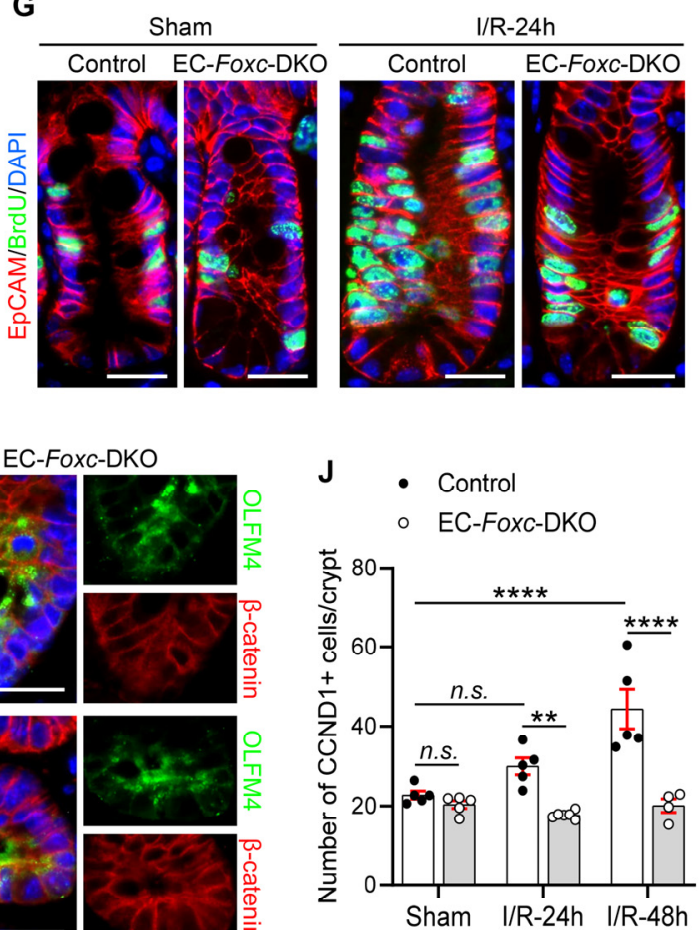

Fig. 2. Characterization of defects in the intestinal mucosa in mice with EC-specific

deletion of Foxc1/2 after I/R. (A) Schematic showing the time of Tamoxifen (Tm) injection, I/R surgery and tissue collection (TC). (B) Representative H\&E staining images of the distal jejunums. The intestinal ischemic injury grading in the Chiu scoring system is indicated by red numbers $(0 \sim 5)$. Scale bars $=100 \mu \mathrm{m}$. Quantification of Chiu Score for $(\mathbf{C})$ Control and EC-Foxc-DKO, (D) Control and EC-Foxc1-KO, (E) Control and EC-Foxc2KO groups. Data are mean $\pm \mathrm{SEM}$, Mann-Whitney $U$ test, $\mathrm{N}=3$ in $\mathrm{C}$ sham groups, $\mathrm{N}=$ 
7 12 in B, C, D I/R-24h groups, ${ }^{*} P<0.05, * * * P<0.001$. (F) Relative mRNA expression of proinflammatory mediators $C o x-2, T N F-\alpha$ and $I L-6$ from intestinal tissue lysates at I/R-3h. Data are mean \pm SEM, Mann-Whitney $U$ test, $\mathrm{N}=6 \sim 11,{ }^{*} P<0.05$, n.s. $=$ not significant. (G) Representative immunostaining images of intestinal crypts labeled with BrdU (proliferative marker, injection performed $2 \mathrm{~h}$ before tissue collection) and EpCAM (epithelial marker) show the proliferation of epithelial cells in crypts. Scale bars $=20 \mu \mathrm{m}$. (H) Quantification of the number of BrdU+ epithelial cells per crypt. Data are mean \pm SEM, Kruskal-Wallis One-way ANOVA test, $\mathrm{N}=3 \sim 6 .{ }^{*} P<0.05$, n.s. $=$ not significant. (I) Representative images of intestinal crypts immunostained with $\beta$-catenin and the intestinal epithelial stem cell (ISC) marker OLFM4. At I/R-48h the nuclear translocation of $\beta$-catenin in ISCs (indicated by arrows) was found in control, whereas it's seldom found in EC-FoxcDKO. Scale bars $=20 \mu \mathrm{m}$. (J) Quantification of the number of CCND1+ epithelial cells per crypt based on the intestinal immunostaining of CCND1 (Fig. S6A). Data are mean \pm SEM, Kruskal-Wallis One-way ANOVA test, $\mathrm{N}=4 \sim 6 .{ }^{*} P<0.01, * * * * P<0.0001$, n.s. $=$ not significant. 

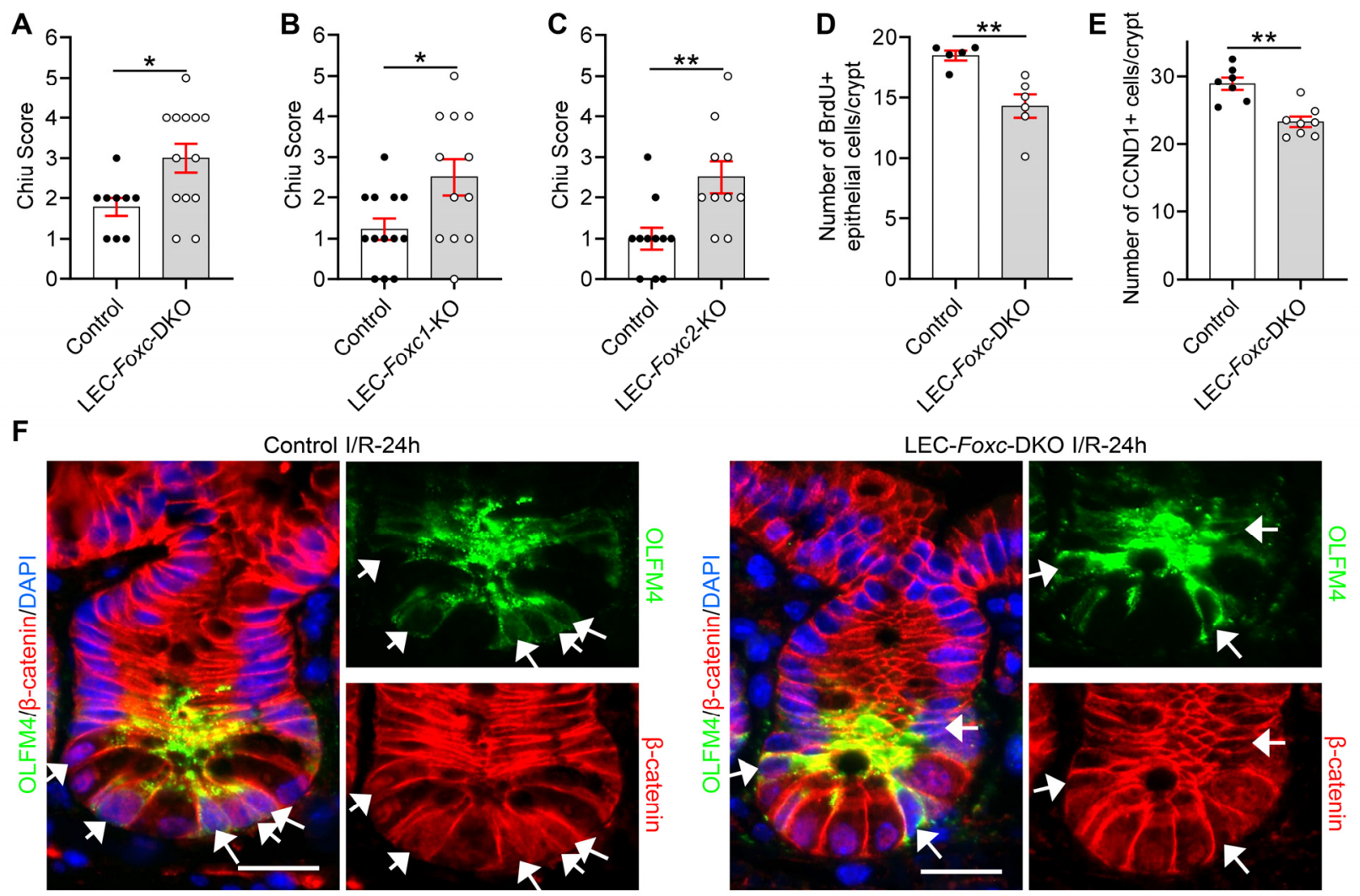

Fig. 3. Characterization of defects in the intestinal mucosa in mice with LEC-specific

deletion of Foxc1/2 after I/R. Chiu Score analysis from H\&E stained distal jejunum $24 \mathrm{~h}$

after I/R for: (A) Control (Foxc1 $\left.{ }^{f / f} ; F_{o x c} 2^{f / f}\right)$ and LEC-Foxc-DKO (Vegfr3$\mathrm{Cre}^{E R T 2} ;$ Foxc $\left.^{f / f} ; F_{\text {oxc }} 2^{f / f}\right)$, (B) Control $\quad\left(\right.$ Foxc $\left.^{f / f}\right)$ and LEC-Foxc1-KO (Vegfr3$\left.\operatorname{Cre}^{E R T 2} ; F_{\text {oxc }} 1^{f / f}\right)$, (C) Control $\left(\right.$ Foxc $\left.^{f f f}\right)$ and LEC-Foxc2-KO (Vegfr3-Cre $\left.{ }^{E R T 2} ; F_{\text {oxc }} 2^{f f f}\right)$.

Data are mean \pm SEM, Mann-Whitney $U$ test, $\mathrm{N}=9 \sim 13, * P<0.05, * * P<0.01$.

Quantification of the number of BrdU+ epithelial cells per crypt in control and LEC-Foxc-

DKO mice at $24 \mathrm{~h}$ after I/R. Data are mean \pm SEM, Mann-Whitney $U$ test, $\mathrm{N}=5 \sim 6$.

$* * P<0.01$. (E) Quantification of the number of CCND1+ epithelial cells per crypt at I/R-

24h from the intestinal immunostaining of CCND1 (Fig. S6B). Data are mean \pm SEM,

Mann-Whitney $U$ test, $\mathrm{N}=7 \sim 8$. ${ }^{* *} P<0.01$. (F) Representative images of crypts 
immunostained with OLFM4 and $\beta$-catenin in control and LEC-Foxc-DKO mice $24 \mathrm{~h}$ after $\mathrm{I} / \mathrm{R}$. The accumulation of $\beta$-catenin in the nuclei of ISCs (indicated by arrows) was found in Control but inhibited in LEC-Foxc-DKO mice. Scale bars $=20 \mu \mathrm{m}$. 

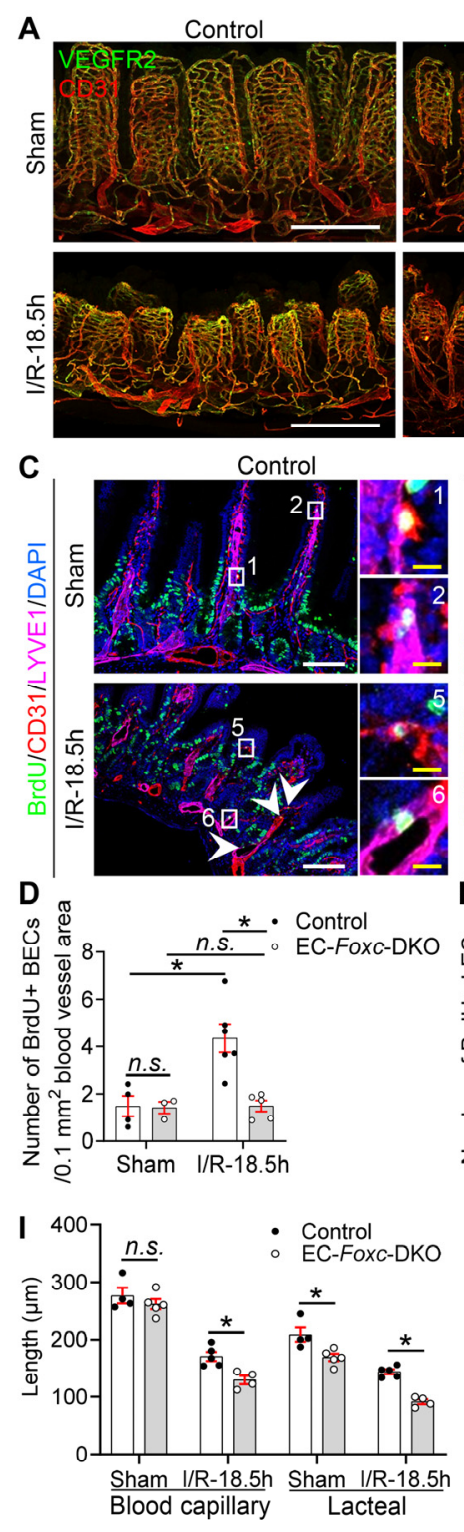

EC-Foxc-DKO
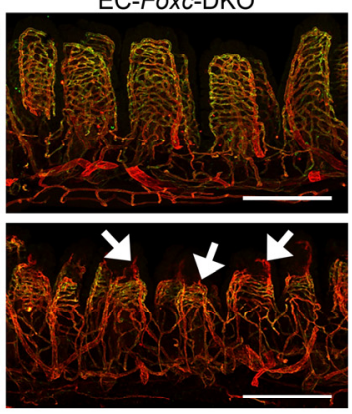

EC-Foxc-DKO
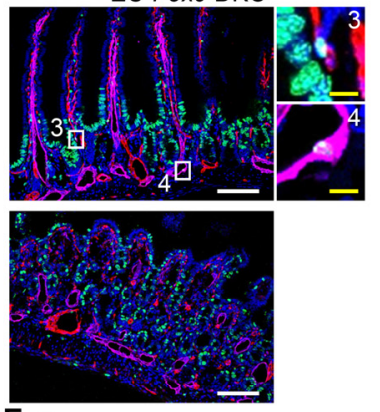

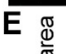

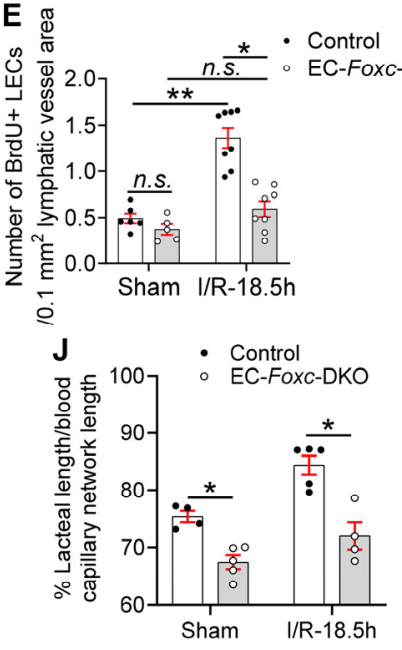

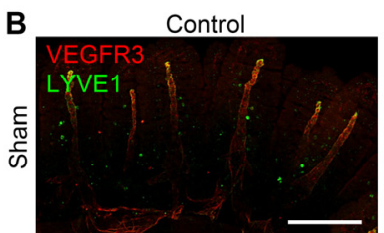
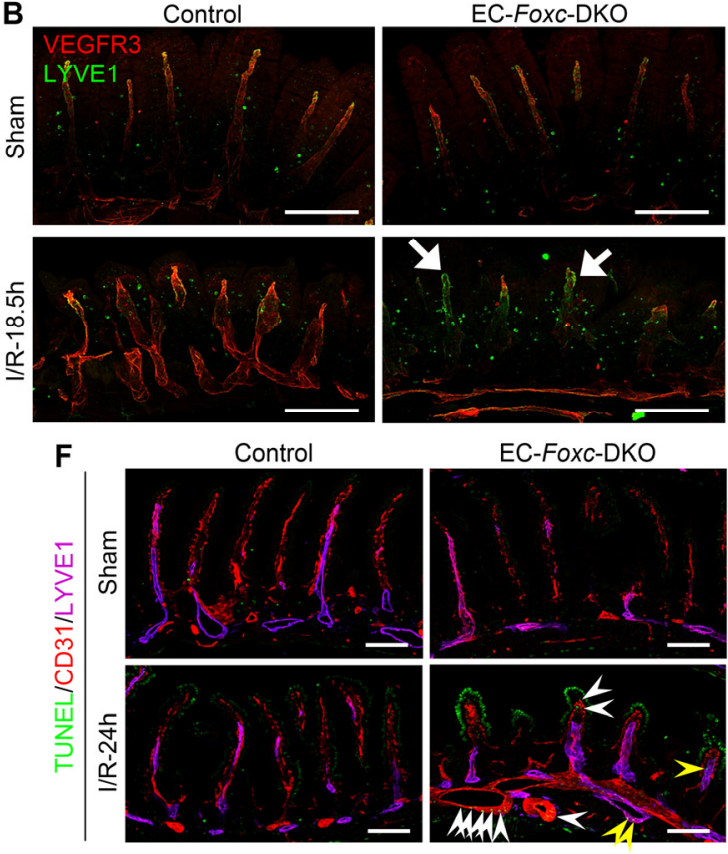

G $\underset{\mathscr{d}}{\mathbb{\infty}} \cdot$ Control

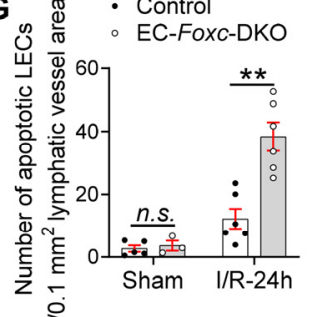

H Control

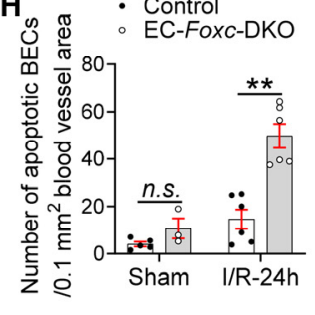

$\mathbf{K}^{\underline{0}}$

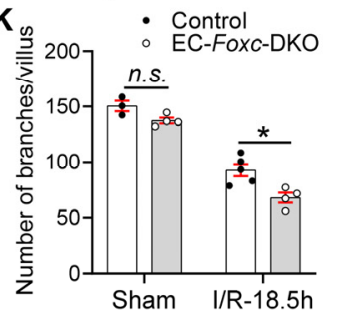

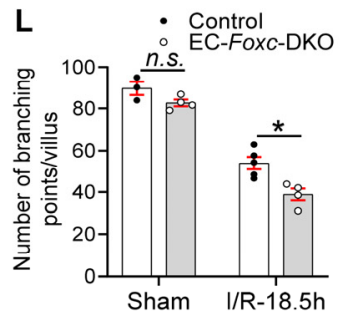

Fig. 4. Defects in vascular regeneration after intestinal $I / R$ injury in EC-Foxc-DKO

mouse. (A) Representative images of intestinal whole-mount VEGFR2/CD31 immunostaining show increased VEGFR2 expression at the angiogenic front of villous blood capillaries in control mice at I/R-18.5h. In EC-Foxc-DKO, the increase of VEGFR2 is inhibited in villous blood vessels and the repair of blood vasculatures is also impaired (arrow). Scale bars $=200 \mu \mathrm{m}$. (B) Representative images of intestinal whole-mount 
VEGFR3/LYVE1 immunostaining. 18.5h after I/R, VEGFR3 is increased in lacteals especially at the lacteal tips in the control but is inhibited in the EC-Foxc-DKO lacteals (arrow). Scale bars $=200 \mu \mathrm{m}$. (C) Representative BrdU/CD31/LYVE1/DAPI immunostaining images of intestinal paraffin sections $(15 \mu \mathrm{m})$ from mice injected with BrdU 18.5h before euthanasia for the analysis of proliferative BECs $(1,3,5)$ and LECs $(2$, $4,6)$ in intestines. Arrow heads show BrdU+ BECs/LECs. White/yellow bars $=100$ or 10 $\mu \mathrm{m}$, respectively. The numbers of BrdU+ BECs (D) and LECs (E) in intestinal mucosa were quantified based on Fig. 4C. Kruskal-Wallis One-way ANOVA test, $\mathrm{N}=3 \sim 8$. (F) Representative TUNEL/CD31/LYVE1/DAPI immunostaining images of distal jejunums for the analysis of apoptotic BECs/LECs (white/yellow arrow heads) in intestinal sections $(15 \mu \mathrm{m})$. Scale bars $=100 \mu \mathrm{m}$. The numbers of apoptotic BECs $(\mathbf{G})$ and LECs $(\mathbf{H})$ were quantified based on Fig. 4F. Mann Whitney $U$ test, $N=3 \sim 6$. The length of blood capillary vasculature and lacteals were measured (I) based on Fig. S7, A and B. The percentage (\%) of the lacteal length/blood capillary network length was calculated $(\mathbf{J})$. The numbers of branches (K) and the branching points (L) of the villous blood vasculatures were counted based on Fig S7C. Mann Whitney $U$ test, $\mathrm{N}=4 \sim 5$. Data are mean $\pm \mathrm{SEM}, * P<0.05$, $* * P<0.01$. n.s. $=$ not significant. 

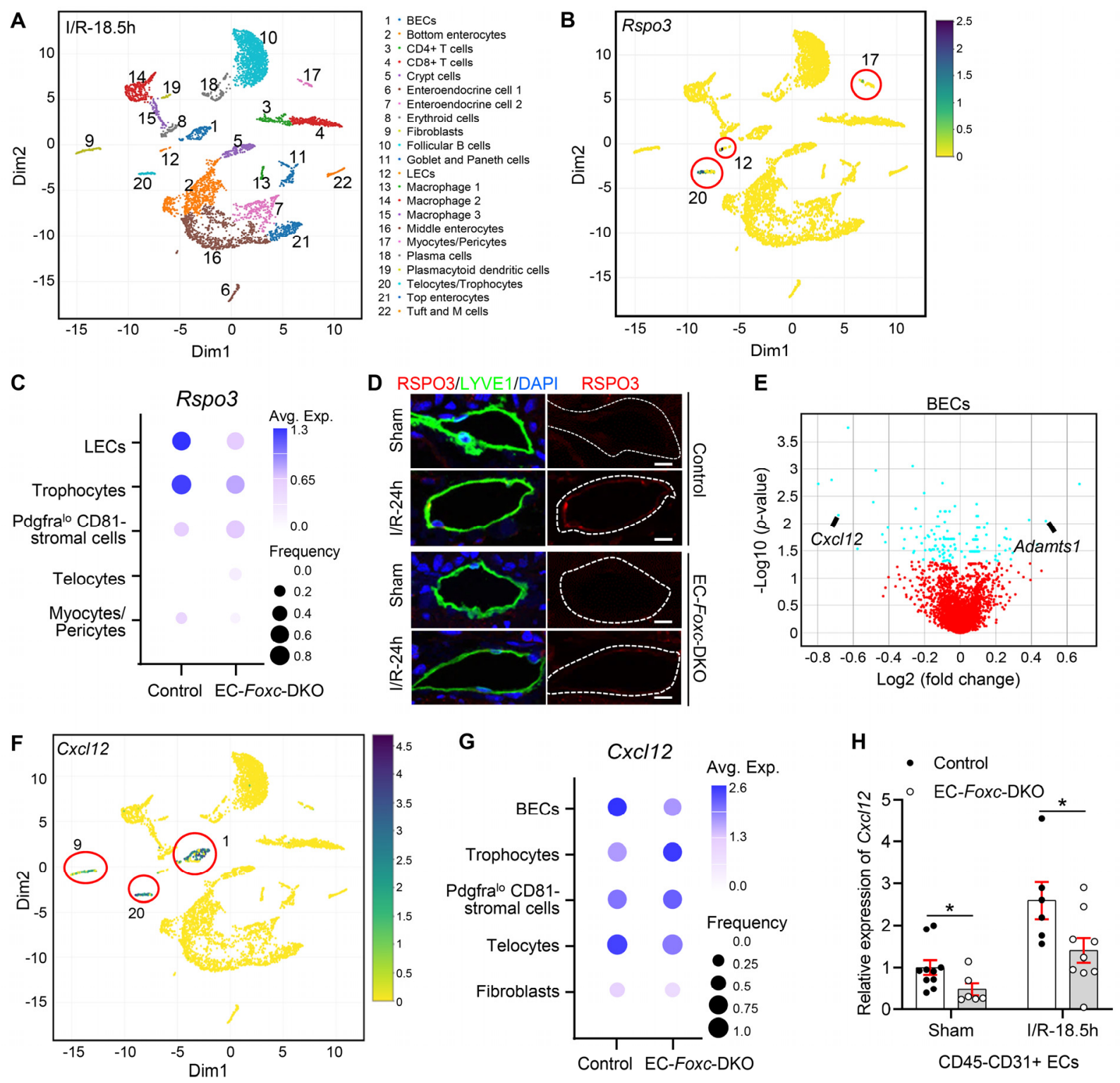

Fig. 5. Single-cell RNA sequencing characterization of the distal jejunum from

Control and EC-Foxc-DKO mice after I/R at 18.5h. (A) Visualization of unsupervised clustering of 22 distinct clusters by UMAP from the distal jejunum of both control and ECFoxc-DKO mice after I/R at 18.5h. (B) UMAP visualization of Rspo3 expression in LECs (12), Myocytes/Pericytes (17), and Telocytes/Trophocytes (20) cell clusters identified in Fig. 5A. (C) Dot plot showing relative expression of Rspo3 in 5 cell clusters identified by 
scRNA-seq. Fill colors represent normalized mean expression levels and circle sizes represent the within-cluster frequency of positive gene detection. (D) Representative images of lymphatic vessels (surrounded by the white dotted lines) in intestinal mucosa labeled with RSPO3/LYVE1. Scale bars $=10 \mu \mathrm{m}$. (E) Volcano plots of differential expression analysis of BECs in Control and EC-Foxc-DKO mice at I/R-18.5h using the MAST model. Blue dots denote genes with significant differential expression, $P<0.05$. (F) UMAP visualization of Cxcl12 expression in BECs (1), fibroblasts (9) and Telocytes/Trophocytes (20) cell clusters identified in Fig. 5A (G) Dot plot showing relative expression of Cxcl12 in 5 cell clusters identified by scRNA-seq. (H) Relative mRNA expression of Cxcl12 in Dynabeads-isolated ECs (CD45-CD31+) from distal jejunum. Data are mean \pm SEM, Mann Whitney $U$ test, $\mathrm{N}=6 \sim 10,{ }^{*} P<0.05$. 
A

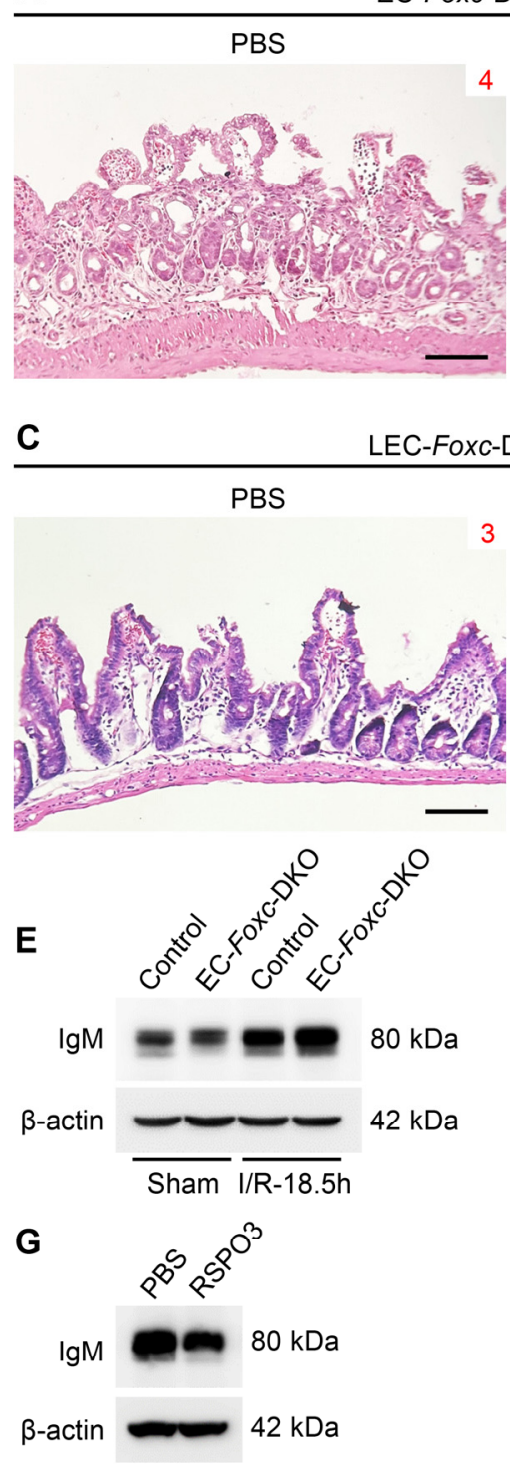

EC-Foxc-DKO I/R-24h

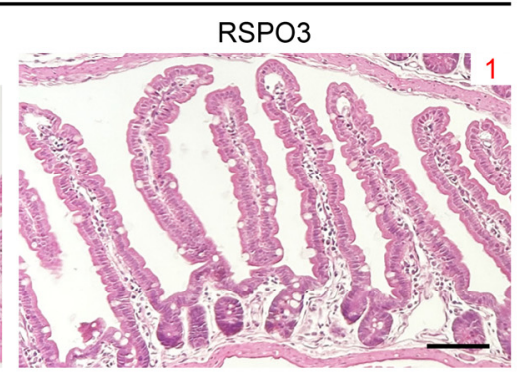

I/R-24h

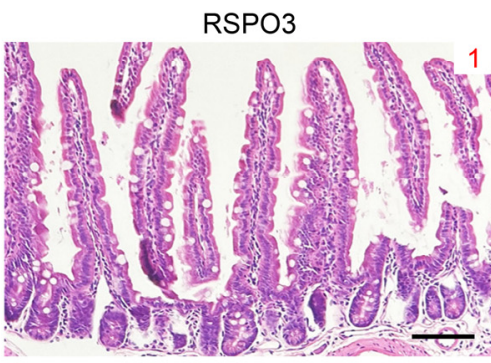

F Control

- EC-Foxc-DKO

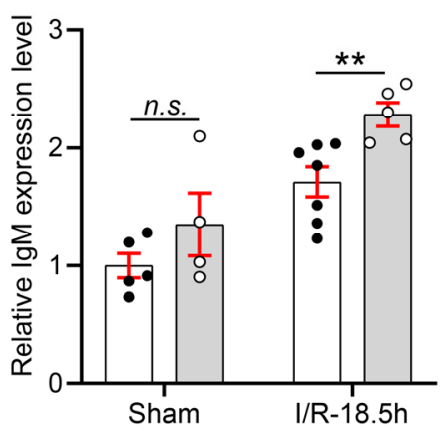

B

EC-Foxc-DKO I/R-24h

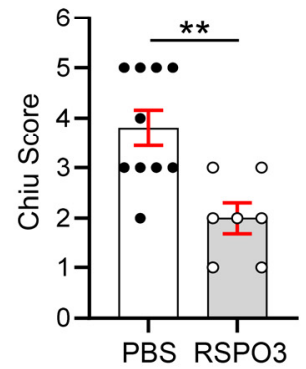

D

LEC-FOXC-DKO I/R-24h

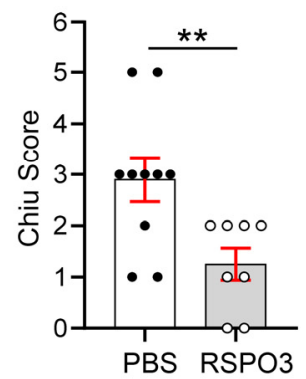

H

EC-Foxc-DKO I/R-18.5h

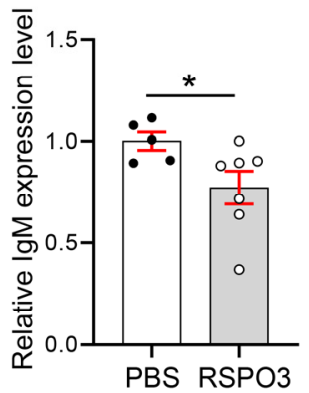

Fig. 6. RSPO3 partially rescues impaired regeneration of intestinal mucosa in ECFoxc-DKO and LEC-Foxc-DKO mice after I/R. In RSPO3 rescue experiment, each mouse was treated with $5 \mu \mathrm{g}$ RSPO3 in $100 \mu \mathrm{L}$ PBS by retro-orbital injection 30 min before ischemia. PBS treated mice were used as control. Representative images of H\&E staining show the rescue effects of RSPO3 in intestinal mucosa in EC-Foxc-DKO (A) and LECFoxc-DKO (C) mouse strains $24 \mathrm{~h}$ after I/R. Red numbers indicate the Chiu scores. Scale 
bars $=100 \mu \mathrm{m}$. Quantification of Chiu Score for RSPO3 rescued (B) EC-Foxc-DKO and (D) LEC-Foxc-DKO intestines 24h after I/R. Data are mean \pm SEM, Mann-Whitney $U$ test $\mathrm{N}=7 \sim 13, * * P<0.01$.Representative Western blots $(\mathbf{E}, \mathbf{G})$ and densitometry measurements $(\mathbf{F}, \mathbf{H})$ show IgM (heavy chain) in intestinal tissue lysates from control and EC-Foxc-DKO mice in sham and after I/R at $18.5 \mathrm{~h}(\mathbf{E}, \mathbf{F})$ as well as IgM (heavy chain) in PBS- and RSPO3- treated EC-Foxc-DKO mice after I/R at 18.5h $(\mathbf{G}, \mathbf{H})$. Bar graphs represent fold change relative to Control sham group. Data are mean \pm SEM, Mann-Whitney $U$ test, $\mathrm{N}=$ 4 7, $* P<0.05, * * P<0.01$. n.s. $=$ not significant. 
A

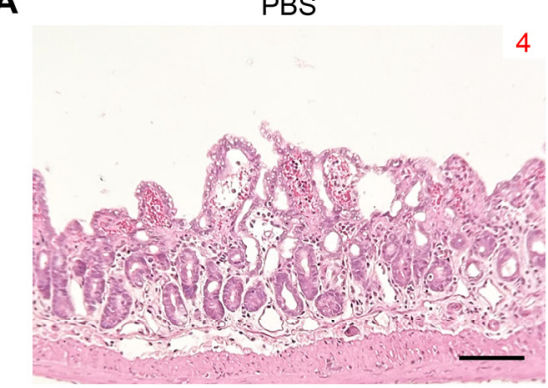

C

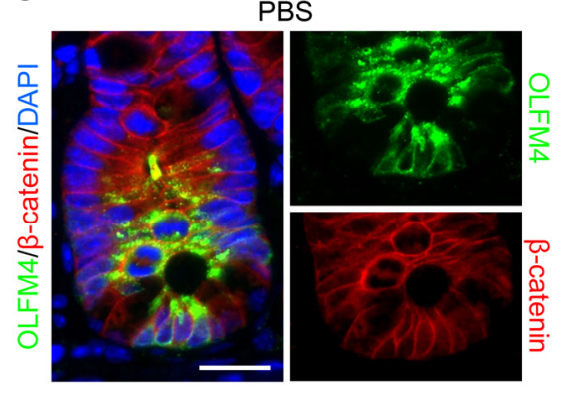

E

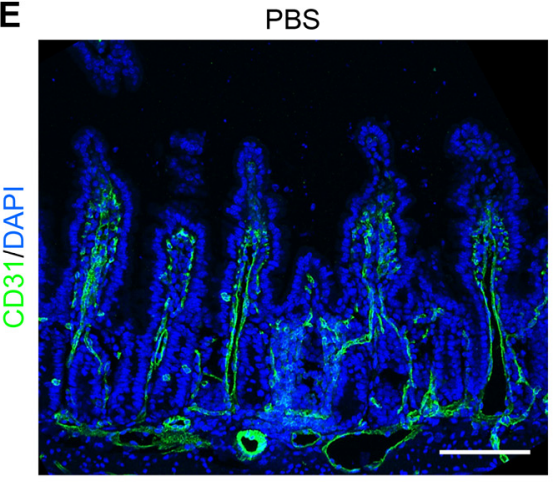

CXCL12

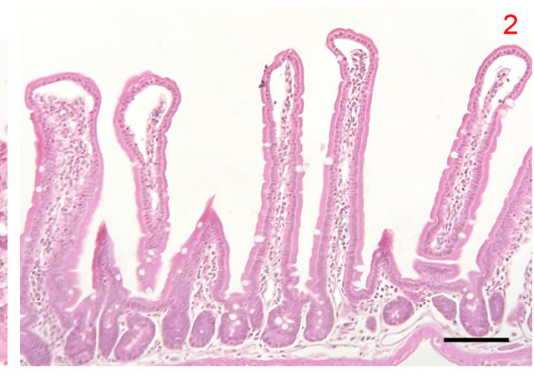

CXCL12

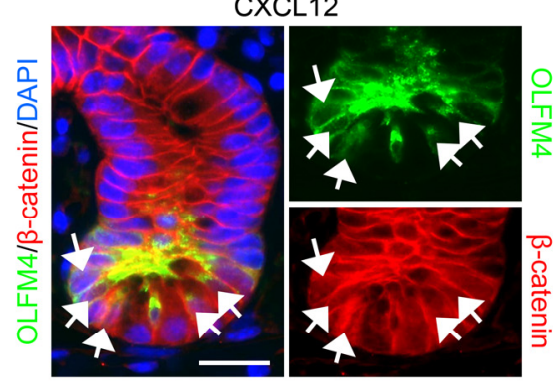

CXCL12

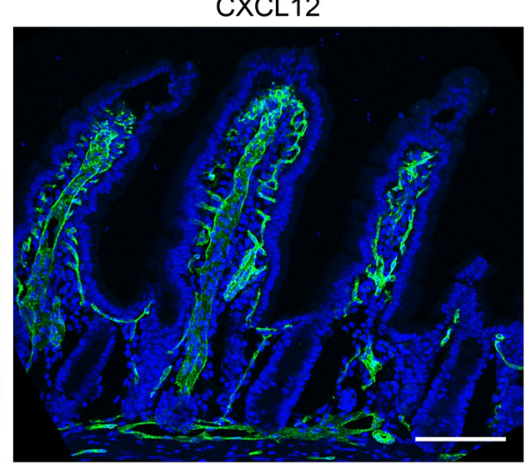

B

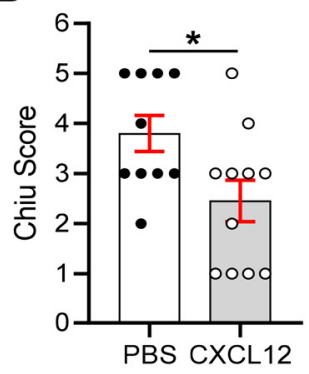

D

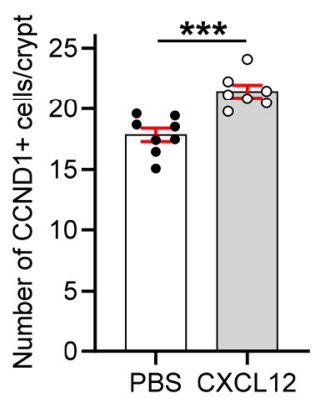

$\mathbf{F}$

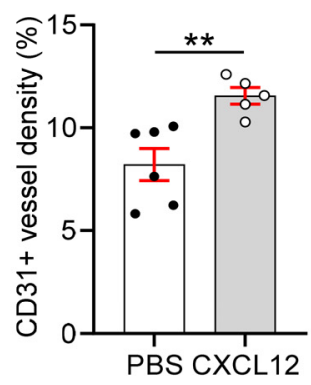

Fig. 7. CXCL12 partially rescues impaired regeneration of intestinal mucosa in EC-

Foxc-DKO mice after I/R. In CXCL12 rescue experiments, mice were treated with $50 \mu \mathrm{g} / \mathrm{kg}$ CXCL12 in PBS by retro-orbital injection $30 \mathrm{~min}$ before ischemia. Mice treated with PBS were used as control. (A) Representative images of H\&E staining show the rescue effects of CXCL12 in intestinal mucosa in EC-Foxc-DKO mice $24 \mathrm{~h}$ after I/R. Red numbers indicate Chiu scores. Scale bars $=100 \mu \mathrm{m}$. (B) Quantification of Chiu Scores for CXCL12-rescued EC-Foxc-DKO mice 24h after I/R. Data are mean \pm SEM, MannWhitney $U$ test, $\mathrm{N}=10 \sim 11,{ }^{*} P<0.05$. (C) Representative images of crypts immunostained 
with OLFM4 and $\beta$-catenin in PBS- and CXCL12-treated EC-Foxc-DKO mice $24 \mathrm{~h}$ after $\mathrm{I} / \mathrm{R}$. The total protein signal of $\beta$-catenin is up-regulated in CXCL12-treated crypts compared with PBS-treated group. The accumulation of $\beta$-catenin in the nuclei of ISCs (indicated by arrows) was found in CXCL12-treated mice but not in PBS-treated mice. Scale bars $=20 \mu \mathrm{m}$. (D) Quantification of CCND1+ epithelial cells per crypt after I/R at 24h. Data are mean \pm SEM, Mann-Whitney $U$ test, $N=7 \sim 8$. $* * * P<0.001$. (E) Representative confocal images of CD31 immunostaining of distal jejunums in PBS- and CXCL12- treated EC-Foxc-DKO mice after I/R at 24h. Scale bars $=100 \mu \mathrm{m}$. (F) Quantification of CD31+ vessel density $(\%=$ total CD31+ vessel area/total intestinal tissue area $\mathrm{x} 100 \%$ ) based on Fig. 7E. Data are mean \pm SEM, Mann Whitney $U$ test, $N=5 \sim 6$. $* * P<0.01$.

\section{Supplementary Materials including:}

Figs. S1 to S10

Tables S1 to S3

Movies S1 to S2 